\title{
Chapter 4 Mapping Conceptual Models to Database Schemas
}

\author{
David W. Embley and Wai Yin Mok
}

\subsection{Introduction}

The mapping of a conceptual-model instance to a database schema is fundamentally the same for all conceptual models. A conceptual-model instance describes the relationships and constraints among the various data items. Given the relationships and constraints, the mappings group data items together into flat relational schemas for relational databases and into nested relational schemas for object-based and XML storage structures.

Although we are particularly interested in the basic principles behind the mappings, we take the approach of first presenting them in Sections 4.2 and 4.3 in terms of mapping an ER model to a relational database. In these sections, for the ER constructs involved, we (1) provide examples of the constructs and say what they mean, (2) give rules for mapping the constructs to a relational schema and illustrate the rules with examples, and (3) state the underlying principles. We then take these principles and show in Section 4.4 how to apply them to UML. This section on UML also serves as a guide to applying the principles to other conceptual models. In Section 4.5 we ask and answer the question about the circumstances under which the mappings yield normalized relational database schemas. In Section 4.6 we extend the mappings beyond flat relations for relational databases and show how to map conceptual-model instances to object-based and XML storage structures. We provide pointers to additional readings in Section 4.7. Throughout, we use an application in which we assume that we are designing a database for a bed and breakfast service. To illustrate all conceptual-modeling features of

David W. Embley

Brigham Young University, Provo, Utah 84602, USA, e-mail: embley@cs.byu.edu

Wai Yin Mok

University of Alabama in Huntsville, Huntsville, Alabama 35816, USA, e-mail: mokw@uah.edu 


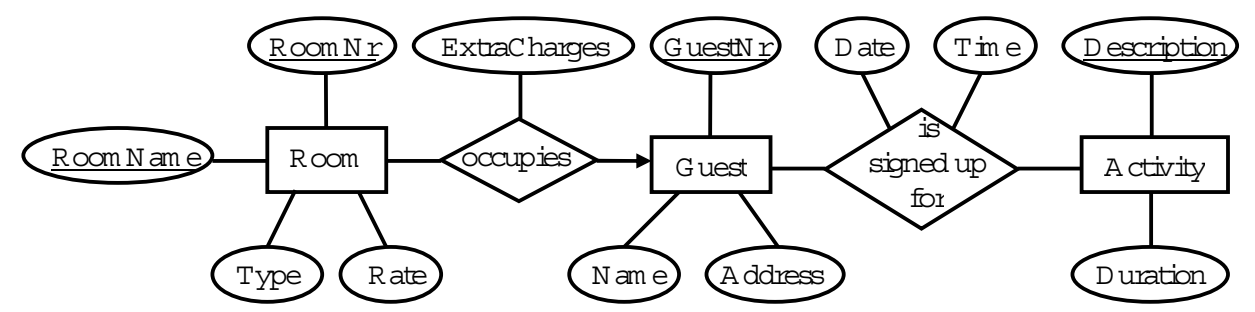

(a) ER Diagram.

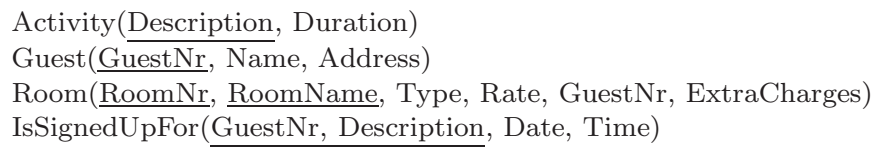

(b) Generated Schemas.

Fig. 4.1 Basic Mappings: ER Diagram and Generated Schemas.

interest, we take the liberty of poetic license in imagining what features might be of interest to the application.

This chapter assumes a solid understanding of several other chapters in this handbook: The Entity-Relationship Model (Chapter 3), The Enhanced EntityRelationship Model (Chapter 4), The Unified Modeling Language (Chapter 5), and Functional Dependencies and Normalization (Chapter 6). We do not extensively discuss any of these topics. We do, however, add enough commentary about these topics to make this chapter reasonably self contained. This chapter also assumes a minimal understanding of Chapter 8 (Relational Data Model), Chapter 10 (SQL), Chapter 14 (Object-Oriented Databases), Chapter 15 (Object-Relational Databases), and Chapter 16 (XML Databases). We make no explanatory comments about these topics.

\subsection{Entity-Relationship Model Mappings}

\subsubsection{Basic Mappings}

We give an ER diagram in Figure 4.1a and the database schema generated from the ER diagram in Figure 4.1b. In our bed and breakfast application, as modeled in Figure 4.1, registered guests occupy rooms and are signed up for activities such as golf, tennis, and horseback riding. Although there may be other occupants of a room in a registered guest's party, in this initial example, we only capture each registered guest (presumably the ones who are paying the bill). Further, in this example we only allow a registered guest's party as a whole to sign up various activities. 
Notationally, each box in Figure 4.1a represents an entity set, e.g., Room, Guest, and Activity. The diamonds with lines connected to entity sets represent relationship sets among the connected entity sets, e.g., occupies and is signed up for. The ovals represent attributes, e.g., RoomNr, Date, and Duration, which may be connected either with entity sets (boxes) or relationship sets (diamonds).

Cardinality constraints for binary relationship sets are one of

1. many-many, indicated by the absence of arrowheads on the lines connecting entity sets and relationship sets, e.g., is signed up for;

2. many-one, indicated by an arrowhead on the one side, e.g., occupies is many-one from Room to Guest, and thus there can be only one registered guest's party occupying a room, although the registered guest's party may occupy one or more rooms;

3. one-many, which is the same as many-one only in the opposite direction, e.g., occupies is one-many from Guest to Room; and

4. one-one, indicated by arrowheads on both sides, e.g., occupies would be one-one if the bed and breakfast had the policy that a guest's party could occupy at most one room.

Cardinality constraints for attributes are many-one from entity set to attribute or from relationship set to attribute. If an entity-set attribute is a key, however, as indicated by underlining the attribute name, then the cardinality is one-one. Thus, for example, Type is many-one from Room to Type so that many rooms can be of the same type (e.g., the bed and breakfast can have several single rooms, several double rooms, and several suites). Room Nr, on the other hand, is a key attribute for Room, and thus each room has one room number and each room number designates one room. Room Name is also a key for Room (each room has a name such as the "Gold Room" or the "Penthouse Suite"). Although rare, relationship sets may also have keys designated by an underline (e.g., a guarantee number for a guest's reservation for a room). Relationship sets, of course, have keys, often a composite of the keys for its related entity sets (e.g., \{Guest Nr, Description\}, which is a composite key ${ }^{1}$ for the relationship set is signed up for). The standard ER model, however, provides no way to directly designate composite keys for relationship sets.

ER Mapping Rule \#1. An entity set $E$ with $n$ key attributes $A_{1}, \ldots, A_{n}$ and $m$ non-key attributes $B_{1}, \ldots, B_{m}$ maps to the relational schema $E\left(\underline{A_{1}}\right.$, $\left.\ldots, A_{n}, B_{1}, \ldots, B_{m}\right)$. The underlines designate keys for the relational schema. If there is only one key, it is the primary key; if there are several keys, one is designated as the primary key. ER Mapping Rule \#1 applies when $E$ is a regular entity set (i.e., not a weak entity set) and has no entity set $E^{\prime}$

\footnotetext{
${ }^{1}$ Here and throughout the chapter "composite key" always designates a minimal key, so that if any of the attributes of the key is removed, the remaining attribute(s) no longer provide the unique identification property of a key.
} 
connected to $E$ by a relationship set that is one-one or is many-one from $E$ to $E^{\prime}$.

ER Mapping Rule \#1 applies to Activity in Figure 4.1a. Activity is a regular entity set (as are all entity sets in Figure 4.1a), and its only connected relationship set is many-many. When we apply ER Mapping Rule \#1 to Activity, since Description is a key attribute and Duration is a non-key attribute, we obtain Activity(Description, Duration), which is the first relational schema in Figure 4.1b. ER Mapping Rule \#1 also applies to Guest, yielding the second relational schema in Figure 4.1b. ER Mapping Rule \#1 does not apply to Room because the connected relationship set occupies is many-one from Room to Guest.

ER Mapping Rule \#2. Let $E$ be a regular entity set with $n$ key attributes $A_{1}, \ldots, A_{n}, m$ non-key attributes $B_{1}, \ldots, B_{m}$, and $p$ many-oneconnected entity sets whose primary keys are $C_{1}, \ldots, C_{p}$ and which have $q$ attributes $D_{1}, \ldots, D_{q}$ associated with the $p$ many-one relationship sets. Assuming $E$ has no one-one-connected entity sets, $E$ maps to $E\left(\underline{A_{1}}, \ldots, \underline{A_{n}}\right.$, $\left.B_{1}, \ldots, B_{m}, C_{1}, \ldots, C_{p}, D_{1}, \ldots, D_{q}\right)$.

ER Mapping Rule \#2 applies to Room in Figure 4.1a. Room has two key attributes (Room Nr and RoomName), two non-key attributes (Type and Rate), and one many-one-connected entity set with a primary key (GuestNr) and with an attribute (ExtraCharges) on its connecting relationship set (occupies). Thus, applying ER Mapping Rule \#2 to Room, we obtain, Room( RoomNr, RoomName, Type, Rate, GuestNr, ExtraCharges), the third relational schema in Figure 4.1. ${ }^{1}$

ER Mapping Rule \#3. Let $E$ and $E^{\prime}$ be two regular entity sets connected by a single one-one relationship set $R$ between them. Let $E$ have $n$ key attributes $A_{1}, \ldots, A_{n}, m$ non-key attributes $B_{1}, \ldots, B_{m}$, and $p$ manyone-connected entity sets whose primary keys are $C_{1}, \ldots, C_{p}$ and which have $q$ attributes $D_{1}, \ldots, D_{q}$ associated with the $p$ many-one relationship sets. Let $E^{\prime}$ have $n^{\prime}$ key attributes $A_{1}^{\prime}, \ldots, A_{n^{\prime}}^{\prime}, m^{\prime}$ non-key attributes $B_{1}^{\prime}, \ldots, B_{m^{\prime}}^{\prime}$, and $p^{\prime}$ many-one-connected entity sets whose primary keys are $C_{1}^{\prime}, \ldots, C_{p^{\prime}}^{\prime}$ and which have $q^{\prime}$ attributes $D_{1}^{\prime}, \ldots, D_{q^{\prime}}^{\prime}$ associated with the $p^{\prime}$ many-one relationship sets. And let $R$ have $r$ attributes $R_{1}, \ldots, R_{r}$. Then, $E, E^{\prime}$, and $R$ together map to the single relational schema $R\left(\underline{A_{1}}, \ldots, \underline{A_{n}}, \underline{A_{1}^{\prime}}, \ldots, \underline{A_{n^{\prime}}^{\prime}}, B_{1}\right.$, $\ldots, B_{m}, B_{1}^{\prime}, \ldots, B_{m^{\prime}}^{\prime}, C_{1}, \ldots, C_{p}, C_{1}^{\prime}, \ldots, C_{p^{\prime}}^{\prime}, D_{1}, \ldots, D_{q}, D_{1}^{\prime}, \ldots, D_{q^{\prime}}^{\prime}, \bar{R}_{1}, \ldots$ $\left.R_{r}\right)$.

ER Mapping Rule \#3 does not apply to the ER model instance in Figure 4.1. It would apply if occupies were one-one, which would mean that a guest's party would occupy one room and could only occupy one room. If occupies were one-one, then we would map Room, Guest, and occupies

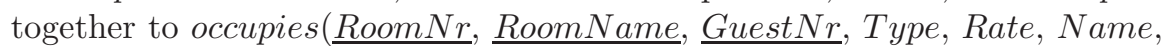
Address, ExtraCharges). Furthermore, there would be no separate schemas

${ }^{1}$ Unless otherwise explicitly stated, the first key listed in a relational schema is is the primary key - Room Nr in this example. 
for Room and Guest schema since both would be entirely included in this occupies schema.

It becomes unwieldy to formally specify further generalizations of ER Mapping Rule \#3. Furthermore, these generalizations seldom arise in practice. The generalizations involve adding more and more entity sets in a one-to-one correspondence with the entity sets already in a one-to-one correspondence. In principle, we just combine together into a single relational schema all the attributes of these entity sets, of their connecting one-one relationship sets, and of their connecting many-one relationship sets (but not their connecting onemany and many-many relationship sets), and all the primary-key attributes of their connecting many-one relationship sets. Unfortunately, however, we have to be careful. Basically the connected one-one relationship sets have to all have mandatory participation; and if there are cycles in the set of entity sets in the one-to-one correspondence, the one-one relationship sets have to all be semantically equivalent. ${ }^{2}$

ER Mapping Rule \#4. Let $R$ be a many-many binary relationship set with attributes $A_{1}, \ldots, A_{n}$. Let $E$ and $E^{\prime}$ be the entity sets connected by $R$, and let $P$ be the primary key attribute of $E$ and $P^{\prime}$ be the primary key attribute of $E^{\prime}$. Then, $R$ maps to $R\left(\underline{P, P^{\prime}}, A_{1}, \ldots, A_{n}\right)$.

ER Mapping Rule \#4 applies to is signed up for, a many-many relationship set whose attributes are Date and Time. Its connected entity sets are Guest and Activity, whose primary keys are respectively Guest Nr and Description. Thus, when we apply ER Mapping Rule \#4, we obtain IsSignedUpFor(GuestNr, Description, Date, Time), which is the last relational schema in Figure 4.1b.

General Principle \#1. In general, mappings of conceptual models to relational schemas are about finding key attributes and composite key attributes and grouping these attributes together into relational schemas along with attributes that directly depend on them. Finding key attributes and composite key attributes is about observing cardinality relationships among attributes (one-one, one-many, many-one, and many-many). Finding directly dependent attributes is about finding attributes that functionally depend on keys, but only on keys within the group of attributes mapped together into a relational schema (i.e., never on some non-key attribute or attribute group, never on a proper subset of a composite key, and never on a combination of a proper subset of a composite key and non-key attributes). Functional dependency arises from cardinality constraints - an attribute $B$ functionally depends on another attribute $A$ if there is a many-one (or one-one) relationship from $A$ to $B$. More generally, an attribute $B$ functionally depends on a set of attributes $A_{1} A_{2} \ldots A_{n}$ if there is a many-one (or one-one) relationship from the $n$-tuples in $A_{1} A_{2} \ldots A_{n}$ to $B$.

General Principle \#2. Graphical instantiations of conceptual models dictate cardinality relationships among attributes. Sometimes the graphical

${ }^{2}$ We refer the interested reader to the additional readings in Section 4.7 for these esoteric mappings. 
instantiations of conceptual models are insufficient to express all needed cardinality relationships. In this case, we express the missing cardinality constraints we need using a formal constraint language when one is defined for the conceptual model or notes in the absence of a defined formal constraint language.

General Principle \#3. The following algorithm generally applies to all conceptual models.

Step 1 Group keys, which may be single-attribute keys or compositeattribute keys, into sets in which the keys in a set are all in a one-to-one correspondence with each other. (In practice, these key sets will often be singleton sets.)

Step 2 In each key set, designate one of the keys (or the only key) to be the primary key for the key set.

Step 3 To each key set, add all directly dependent non-key attributes, plus, from among other key sets, the attributes of all directly dependent primary keys.

Step 4 For each group of attributes formed in Step 3, select a name and form a relational schema. (Name selection is often obvious. Since keys are for entity sets or relationship sets, we typically use the entity-set name or the relationship-set name.)

If we apply General Principle \#3 to the ER diagram in Figure 4.1a, Step 1 yields the set of key sets: $\{\{$ Description $\},\{G u e s t N r\},\{R o o m N r$, RoomName $\},\{$ Guest $N r$ Description $\}$ \}. ${ }^{1}$ In Step 2 we designate Room Nr as the primary key for the key set $\{R o o m N r$, RoomName $\}$. All other key sets are singleton sets and thus each key in these singleton sets is a primary key. In Step 3 we group attributes, and in Step 4 we select names for these attribute groups and form relational schemas. For the key set $\{$ Description\}, the only directly dependent attribute is Duration. Hence, we add it, yielding (Description, Duration). Based on the diagram in Figure 4.1a, the obvious name for this attribute group is Activity. Thus, Activity(Description, Duration) becomes the relational schema for the key set $\{$ Description $\}$. This is the first relational schema in Figure 4.1b. The key set $\{$ Guest $N r\}$ has two directly dependent attributes: Name and Address. Thus, with the addition of the obvious schema name, Guest(GuestNr, Name, Address) becomes the relational schema for the key set $\{$ Guest $N r\}$. This is the second relational schema in Figure 4.1b. The key set $\{R o o m N r$, Room Name $\}$ has three directly dependent non-key attributes: Type and Rate from the entity set Room and ExtraCharges since it is an attribute of occupies, the many-one relationship set from Room to Guest. From among the other key sets, Guest Nr

\footnotetext{
1 Here, we make use of the common set notation in the relational database literature that lets a sequence of attribute names designate a set. Thus, \{Guest $N r$ Description $\}$ is a key set with a single composite key consisting of two attributes whereas Room $\mathrm{Nr}$, RoomName\} is a key set with two keys.
} 
is the only primary key directly dependent on Room Nr. ${ }^{1}$ Thus, with the addition of the obvious schema name, Room (RoomNr, Room Name, Type, Rate, GuestNr, ExtraCharges) becomes the relational schema. This is the third relational schema in Figure 4.1b. Finally, for \{Guest Nr Description\}, which is the key set for the relationship set is signed up for, the only directly dependent attributes are Date and Time. ${ }^{2}$ Thus, with the addition of the obvious schema name, IsSignedUpFor (GuestNr, Description, Date, Time) becomes the relational schema.

Observe that ER Mapping Rules \#1-\#4 are simply special cases of the general principles written specifically for the ER model. ER Mapping Rule \#1 gathers together key attributes and non-key attributes from a single regular entity set that has no connecting functionally dependent entity sets. ER Mapping Rule \#2 gathers together key attributes and non-key attributes from a single regular entity set along with directly functionally dependent attributes that are not keys and the attributes of a primary key from each entity set functionally dependent on the single regular entity set for which we are constructing the relational schema. ER Mapping Rule \#3 and its generalizations gather together all attributes from all entity sets in a one-to-one correspondence, plus all attributes connected to any of the relationship sets forming the one-to-one correspondence, plus all primary key attributes of all the entity sets connected by a many-one relationship set from any one of the entity sets in a one-to-one correspondence, plus all attributes of these many-one relationship sets. ER Mapping Rule \#4 identifies the special case when the composite key for a relationship set consists of primary key attributes of its connecting entity sets. The attributes from these two primary keys along with any attributes connected to the relationship set form the relational schema.

\subsubsection{Complex Key Attributes}

Key identification is a central component of mapping conceptual models to database schemas. Conceptual models commonly provide a direct way to identify keys via diagramming conventions. For the ER model, it is common to underline key attributes for entity sets as Figure 4.1a shows. This convention along with cardinality constraints imposed over relationship sets provides

\footnotetext{
${ }^{1}$ Note that although Name and Address functionally depend on Room Nr and also on Room Name, they do not directly functionally depend on either Room Nr or RoomName because they functionally depend on Guest $N r$, which is not a key attribute for the key set $\{$ Room Nr, RoomName\} for which we are building a relational schema.

2 Note that Duration functionally depends on Description, but since Description is a proper subset of GuestNr Description, we exclude Duration. Similarly, we exclude Name and Address since GuestNr is a proper subset of GuestNr Description.
} 

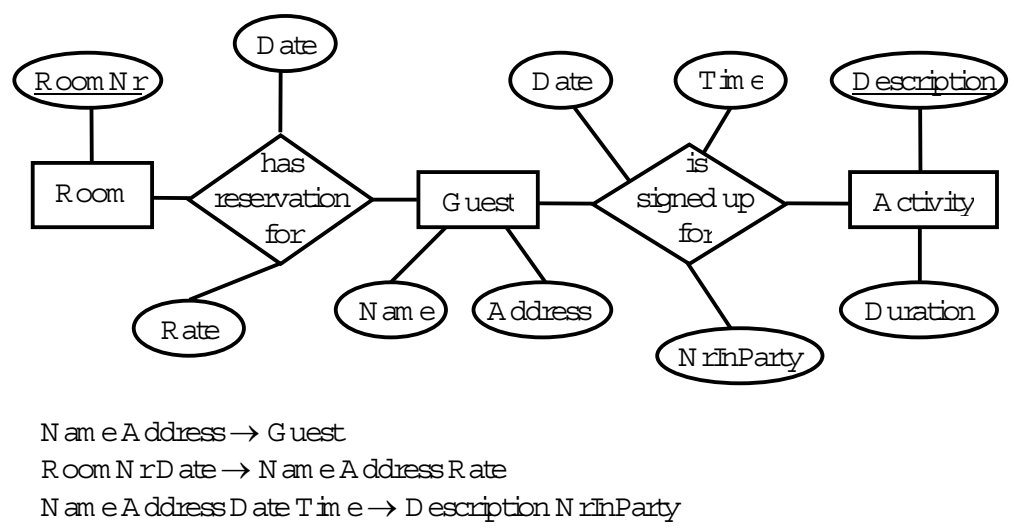

(a) ER Diagram.

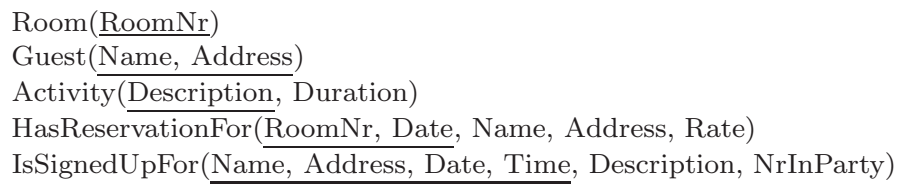

(b) Generated Schemas.

Fig. 4.2 Mappings for Complex Key Attributes: ER Diagram and Generated Schemas.

sufficient information for identifying many keys for entity and relationship sets - indeed most keys in practice.

ER diagramming conventions, however, are not sufficient to allow us to identify all keys. Two common cases are (1) composite keys for entity sets and (2) keys for relationship sets not derivable from information about entity-set keys coupled with relationship-set cardinality constraints. The ER diagram in Figure 4.2a. gives examples of these two cases.

1. The entity set Guest in Figure 4.2a has no key attribute. Neither Name alone nor Address alone uniquely identifies a guest. Many different people with the same name may make reservations, and we may wish to make it possible for different people with the same address to make reservations. Name and Address together, however, may uniquely identify Guest, and we may designate that this must hold in our database so that Name Address becomes a composite key. If no diagramming convention or other formal convention provides a way to designate composite keys for an entity set, ${ }^{1}$ it is best to record this information in a note. In our notes here, we use standard functional dependency (FD) notation, allowing both attribute names and entity-set names to appear as components of left-hand

1 There are many ER variants, and some have conventions to designate composite keys for entity sets (e.g., a connecting line among underlined attributes of an entity set). 
and right-hand sides of FDs. To say that Name Address is a key for Guest, we write the FD Name Address $\rightarrow$ Guest as Figure 4.2a shows.

2. Since only one guest's party can occupy a room on any given date, Room Nr and Date uniquely identify a reservation. Thus Room Nr and Date constitute the composite key for the many-many relationship set has reservation for. This is contrary to the usual convention, which would have the key consist of the primary keys from the connecting entity sets. It is easy to see, however, that Room Nr Name Address cannot be the key because it would allow multiple parties to occupy the same room on the same day. We designate the key for a relationship set by including all the primary-key attributes of all connecting entity sets and all the attributes of the relationship set in an FD. Thus, for example, we write Room Nr Date $\rightarrow$ Name Address Rate as Figure 4.2a shows. Similarly, we write Name Address Date Time $\rightarrow$ Description NrInParty to designate Name Address Date Time as a composite key for is signed up for, i.e., a guest's party can only sign up for one activity at a given time on a given date.

Figure $4.2 \mathrm{~b}$ shows the relational schemas generated from Figure 4.2a. We obtain these relational schemas by following the algorithm in General Principle \#3. In Step 1 we generate keys for each of the entity sets and each of the many-many relationship sets. This step yields the set of key sets $\{\{$ Name Address\}, $\{$ Description $\},\{$ RoomNr Date $\},\{$ Name Address Date Time $\}$, $\{R o o m N r\}$. Since each key set has only one key, it becomes the primary key called for in Step 2. Next we add all directly dependent non-key attributes and needed directly dependent primary-key attributes as called for in Step 3 and select names as called for in Step 4. The result consists of the relational schemas in Figure 4.2b. ${ }^{2}$

ER Mapping Rule \# $\mathbf{1}^{\prime}$, ER Mapping Rule \# $\mathbf{2}^{\prime}$, and ER Mapping Rule $\# \mathbf{3}^{\prime}$. The mapping rules for entity sets with possible composite keys are straightforward generalizations of ER Mapping Rules \#1-\#3. Their formal statement, however, is quite complex. Basically, instead of just simple singleattribute keys, we must also allow for composite keys. Thus, for example, for ER Mapping Rule \#1' (the generalization of ER Mapping Rule \#1), we include all attributes that are keys, all attributes that are parts of composite keys, and all non-key attributes in the generated relational schema. It is possible, though rare, that composite keys overlap. For example, if we had kept Room Name from Figure 4.1a in our example for Figure 4.2a, we would also have the composite key Room Name Date grouped with the composite key Room Nr Date. In this case, the mapping rule should only generate three

${ }^{2}$ Whether we should keep a relation for Room here is an interesting question. Observe that its data may be completely recorded in the relation HasReservationFor. If so, we can discard it. In our application, however, it is possible (even likely) that there is no current reservation for some of the rooms. Thus, to preserve all room numbers in our database, we keep it. 
attributes (not four) for these two composite keys. The single occurrence of Date in the relational schema would be part of both composite keys.

ER Mapping Rule \# $\mathbf{4}^{\prime}$. For any relationship set, we gather together all attributes constituting primary keys of all related entity sets plus all attributes of the relationship set. We then determine, from among these attributes, which attributes and attribute sets are keys. This becomes the relational schema for the relationship set, except in two special cases having to do with ER Mapping Rules $\# 2^{\prime}$ and $\# 3^{\prime}$. If the primary key of one and only one of the connected entity sets $E$ of the relationship set $R$ is a key for $R$, then as part of Mapping Rule \# $2^{\prime}$, all the attributes of the relational schema for $R$ become part of the relational schema for $E$; there is no separate relational schema for $R$. If the primary keys of two or more of the connected entity sets $E_{1}, \ldots, E_{n}$ of the relationship set $R$ is each a key for $R$, then as part of ER Mapping Rule $\# 3^{\prime}$, all the attributes from the relational schemas for $E_{1}, \ldots, E_{n}$, and $R$ are all combined to form a single relational schema for the database.

\subsubsection{Recursive Relationship Sets 6 Roles}

To be understood, recursive relationship sets require roles. Figure 4.3a shows an example. The role Connecting Room helps us understand that the relationship set denotes adjoining rooms with a doorway between them: a Room is connected with a Connecting Room.

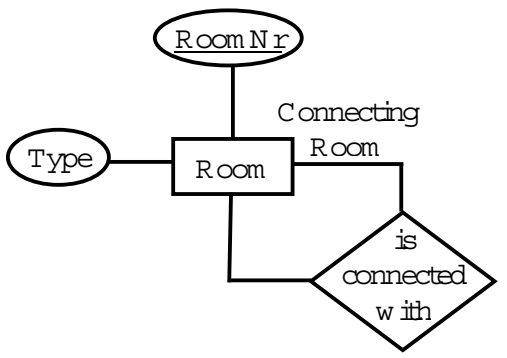

(a) ER Diagram.

Room( $\underline{\text { RoomNr}}$, Type)

IsConnectedWith(RoomNr, ConnectingRoomNr)

(b) Generated Schemas.

Fig. 4.3 Mappings for Roles: ER Diagram and Generated Schemas.

Roles also help us choose attribute names for recursive relationship sets. We map a recursive relationship set to a relational schema in the same way we map regular relationship sets to a relational schema. One-one and many-one 
recursive relationship sets become part of the relational schema for the entity set, and many-many recursive relationship sets become relational schemas by themselves. In all cases, however, there is one difference - we must rename one (or both) of the primary-key attributes. Because the regular mapping for a recursive relationship set would make the primary key of the entity set appear twice, and since a relational schema cannot have duplicate attribute names, we must rename one (or both) of them. The role helps because it usually gives a good clue about what one of the names should be. As Figure 4.3b shows, we map the many-many recursive relationship set to the relational schema with attribute names Room $N r$ and ConnectingRoom $N r$.

We can also use roles in this same way even when a relationship set is not recursive. For example, we could have added a role Occupied Room to the Room side of occupies in the ER diagram in Figure 4.1a. In this case we could have generated Room(OccupiedRoomNr, OccupiedRoom Name, Type, Rate, Guest Nr, ExtraCharges) in which OccupiedRoom Nr replaces Room Nr and OccupiedRoomName replaces RoomName in the relational schema in Figure 4.1b. Using the role name in this way is optional, but may be useful for distinguishing the roles attributes play when we have more than one relationship set between the same two entity sets. For example, we could have also had has reservation for in addition to occupies as a relationship set between Room and Guest in Figure 4.1a.

\subsubsection{Weak Entity Sets}

A weak entity set is an entity set with no key among its directly associated attributes. If we augment our bed and breakfast example to a chain of bed and breakfast establishments as Figure 4.4a shows, the room number no longer uniquely identifies a room. Every bed and breakfast in the establishment likely has a Room \#1 and a Room \#2 and probably more. Thus, Room has no key among its directly associated attributes, and it becomes a weak entity set.

In an ER diagram, we designate a weak entity set by enclosing the name of the weak entity set in a double box. In addition, we add a special relationship set connecting the weak entity set to the entity set on which the weak entity set depends for its key. We designate these special relationship sets by a double diamond and by adding an arrowhead on the side connecting to the entity set on which the weak entity set depends. Adding the arrowhead is appropriate since there is always a many-one relationship from the weak entity set to the entity set on which the weak entity set depends. The ER model does not, by the way, preclude the entity set on which a weak entity set depends from itself being a weak entity set.

Figure 4.4a is an ER diagram showing the three situations in which we normally find weak entity sets. Weak entity sets typically arise (1) when there is 


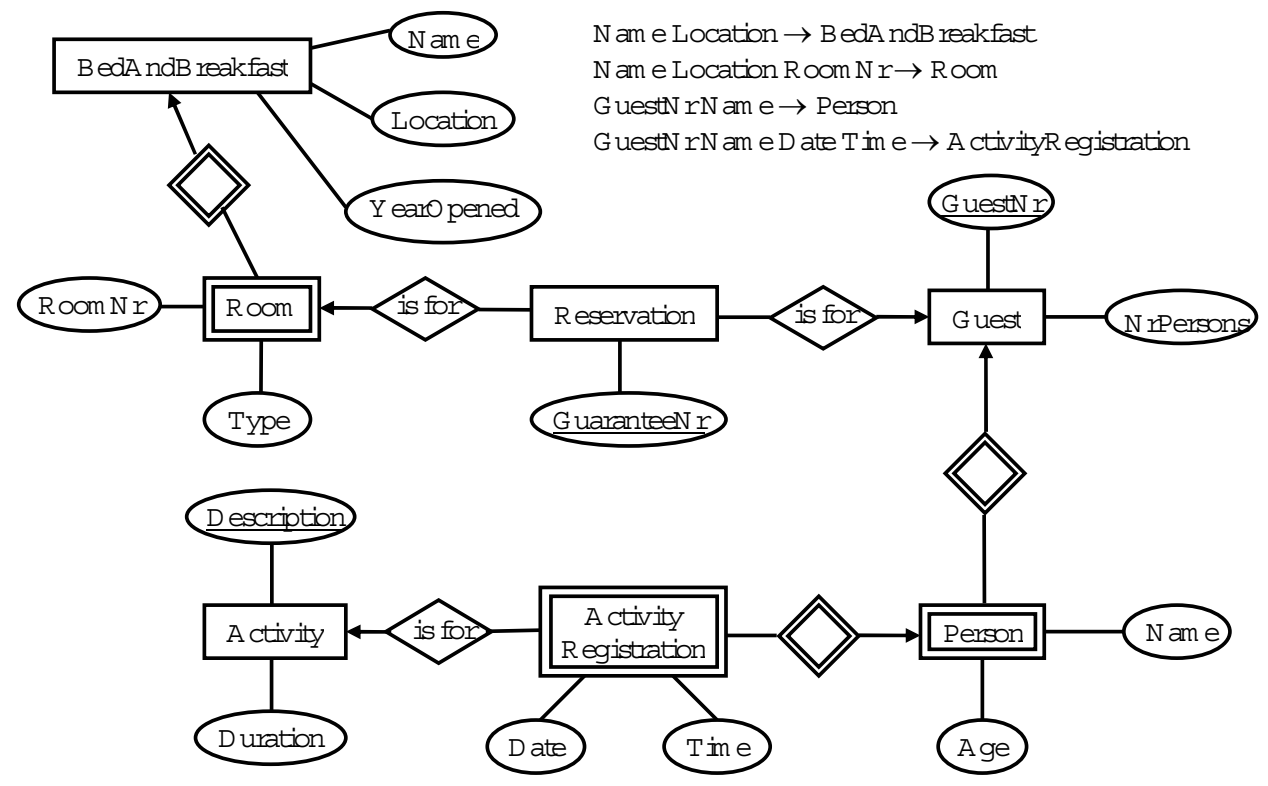

(a) ER Diagram.

BedAndBreakfast(Name, Location, YearOpened)

Room(Name, Location, RoomNr, Type)

Reservation(GuaranteeNr, Name, Location, RoomNr, GuestNr)

Guest(GuestNr, NrPersons)

Person (GuestNr, Name, Age)

Activity(Description, Duration)

ActivityRegistration(GuestNr, Name, Date, Time, Description)

(b) Generated Schemas.

Fig. 4.4 Mappings for Weak Entity Sets: ER Diagram and Generated Schemas.

an organizational subdivision (e.g., Room is an organizational division of the BedAndBreakfast chain), (2) when one entity depends on the existence of another (e.g., for the bed and breakfast database, Person depends on the existence of a registered Guest), and (3) when we wish to view relationship sets as entity sets (e.g., an Activity Registration rather than just a relationship set between Person and Activity). ${ }^{1}$

The general principles tell us how to map weak entity sets to relational schemas. We first identify keys. In every case in which we find a weak entity set, the identity of entities in the weak entity set depends on the key for some other one or more entity sets. The identity of a room depends on the bed and breakfast in which the room is located. The key for BedAndBreakfast,

\footnotetext{
${ }^{1}$ Note, by the way, that the entity set Reservation is not weak, even though it is certainly a relationship set we view as an entity set. When we turned it into an entity set, we gave it a key, GuaranteeNr, so that it did not become a weak entity set.
} 
which for our example is the composite key Name Location, ${ }^{2}$ plus a Room Nr uniquely identify a room. Thus, the key for Room is the composite of all three attributes, namely, Name Location RoomNr. For Person, names are not unique identifiers, but are usually unique within a family, which often constitutes a registered guest's party. In our example, we require unique names within a registered guest's party, and thus the key for the weak entity set Person is the composite of GuestNr and Name (of Person). A person can sign up for only one activity at a given time on a given date. Thus, to uniquely identify an ActivityRegistration, we need the key of Person, which is GuestNr Name, as well as Date and Time to all be part of the key. Thus, the composite key for ActivityRegistration is GuestNr Name Date Time.

After identifying keys for a weak entity set (and designating one to be the primary key in case there are several), we add all directly dependent non-key attributes and directly dependent primary-key attributes. We then choose a name - usually the name of the weak entity set - and form a relational schema. Figure $4.4 \mathrm{~b}$ shows the result for the ER diagram in Figure 4.4a. For the weak entity set Room, the only directly dependent attribute is Type. Thus, since the key is the composite Name Location Room Nr, the relational schema is Room (Name, Location, Room Nr, Type), the second relational schema in Figure 4.4b. Similarly, for Person, since Age is the only directly dependent attribute, the relational schema for the weak entity set is Person(GuestNr, Name, Age). For the weak entity set ActivityRegistration, Description is a directly dependent primary-key attribute. Thus, since the composite key is GuestNr Name Date Time, we generate the last relational schema in Figure 4.4b.

ER Mapping Rule \# . Let $W$ be a weak entity set, let $E$, entity set on which $W$ depends ( $E$ may itself be weak), and let $F_{1}, \ldots, F_{m}$ be the $m$ other entity sets (if any) in a many-one relationship from $W$ to $F_{i}(1 \leq$ $i \leq m)$. Form a relational schema called $W$ with attributes consisting of all the attributes of $W$, the primary-key attribute(s) of $E$, all the primarykey attributes of $F_{1}, \ldots, F_{m}$, and all the attributes (if any) of the many-one relationship sets from $W$ to each $F_{i}(1 \leq i \leq m)$. From among the attributes, determine the keys for $W$ and designate one as the primary key. Each key of $W$ is formed from by adding one or more attributes of $W$ to the primary key for $E .^{3}$

2 Location is meant to be a simple city or town or other designated place. Several bed and breakfast establishments can be in the same location (e.g., Boston), but each establishment in the same location must have a different name. Thus, Name Location $\rightarrow$ BedAndBreakfast.

3 Typically, as in our examples here, $W$ has only one key. But, for example, if we also had Room Name for the weak entity set Room, we would have a second key for Room, namely Name Location Room Name. 


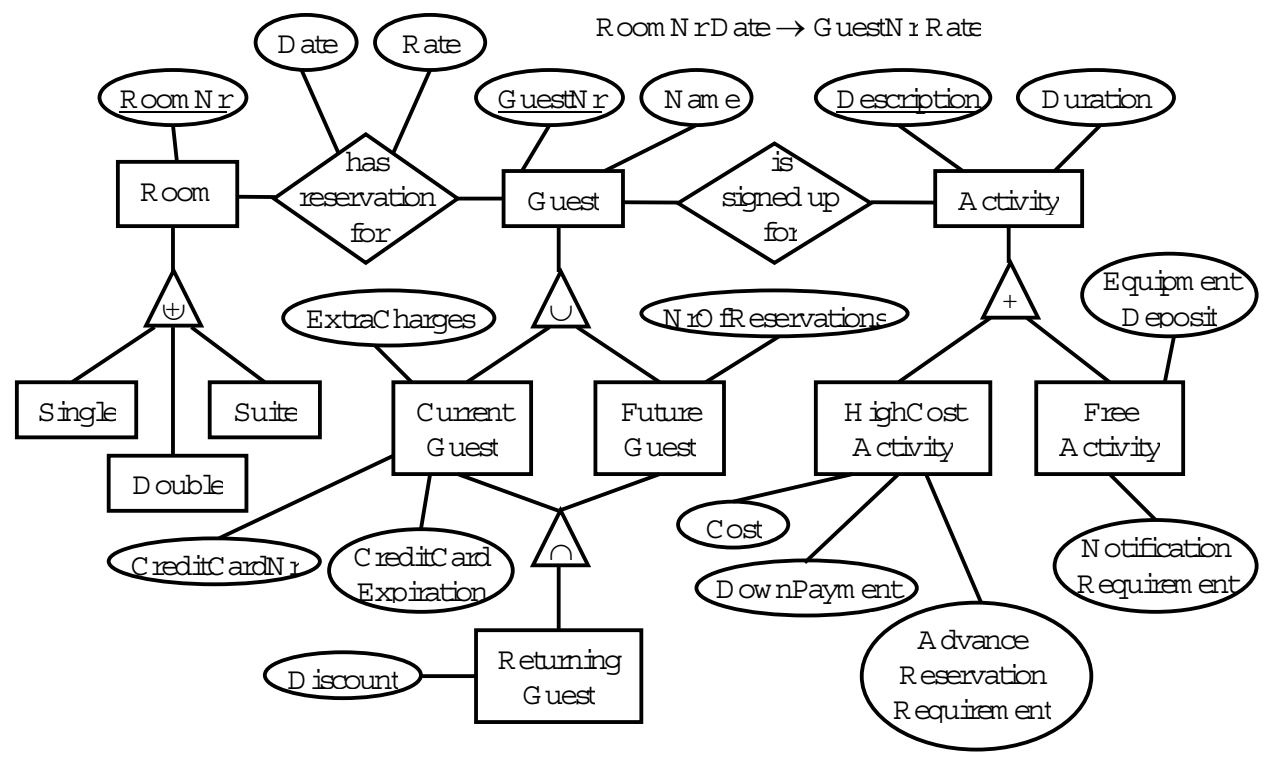

(a) ER Diagram.

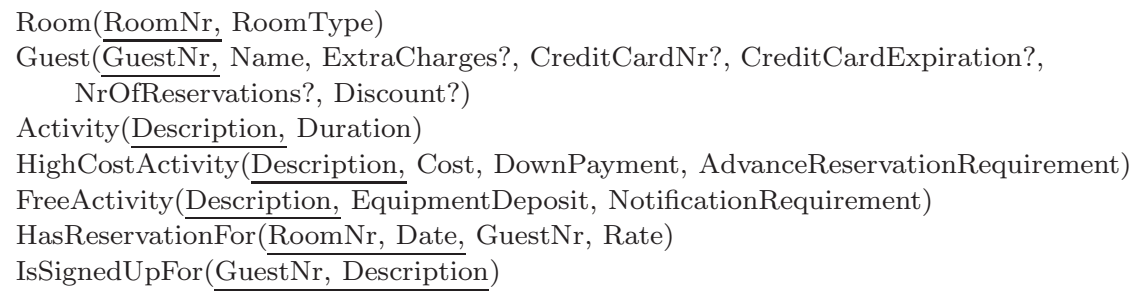

(b) Generated Schemas.

Fig. 4.5 Mappings for ISA Hierarchies: ER Diagram and Generated Schemas.

\subsection{Extended Entity-Relationship Model Mappings}

Extended ER models include generalization/specialization or ISA hierarchies, with possible union, disjoint, overlapping, partition, and intersection constraints. They also include compound attributes, multi-valued attributes, computed attributes, and designations for mandatory and optional participation. In this section, we consider mappings for each of these extensions. 


\subsubsection{ISA Mappings}

Figure 4.5a shows an ER diagram with several ISA constructs. Graphically, triangles denote ISA constructs, which fundamentally designate entity sets as subsets and supersets. ${ }^{1}$ An entity in a subset entity set is also an entity in its superset entity set - thus the "ISA" designation. The apex of a triangle connects to superset entity sets, and the base connects to subset entity sets. In Figure 4.5a both CurrentGuest and FutureGuest are subsets of Guest, and ReturningGuest is a subset of both CurrentGuest and FutureGuest. We can constrain the subsets by placing symbols in the triangles: $\cap$ for intersection, $\cup$ for union, + for mutual-exclusion, and $\uplus$ for partition. An intersection constraint requires that the subset entity set be exactly the intersection of the superset entity sets (e.g., in Figure 4.5a ReturningGuest is exactly the intersection of CurrentGuest and FutureGuest). Without the intersection constraint (triangle with no special constraint inside it) the subset entity set could be a proper subset of the intersection. Union requires that the superset is exactly the union of the subsets (e.g., Guest is defined to be exactly the union of those guests who are currently at the bed and breakfast and those who will be future guests). Mutual-exclusion requires that the subsets pairwise have an empty intersection (e.g., HighCostActivity and FreeActivity have an empty intersection). Partition requires both union and mutual-exclusion (e.g., a room must be a single room, a double room, or a suite).

Figure 4.5b shows the relational schemas generated from the ER diagram in Figure 4.5a. For ISA hierarchies, there are three basic mappings, which we label as ER Mapping Rules \#6.1-\#6.3. (Combinations over multiple-level hierarchies are also possible.)

ER Mapping Rule \#6.1. Make a relational schema for all entity sets in the hierarchy. Although not always best, this is the most straightforward mapping for ISA hierarchies. The mapping for an entity set in an ISA hierarchy that has no generalization is the same as the mapping for any entity set. The mapping for a specialization is also the same except that the primary-key attribute(s) of the generalization $(\mathrm{s})^{2}$ are also added to the relational schema. In general any key for the specialization can be the primary key. Normally, however, there will be only one key, the inherited primary key. In Figure 4.5

\footnotetext{
${ }^{1}$ In this context, we call subset entity sets "specialization entity sets" or just "specializations" and call superset entity sets "generalization entity sets" or just "generalizations."

2 Since all specializations in an ISA hierarchy are subsets of their generalizations, entities in the specializations inherit their identity from their generalization(s). In most common cases there is only one generalization. When a specialization has more than one generalization, it inherits its identity from all generalizations. Often, however, all generalizations have the same identifying attribute inherited from some root generalization. ReturningGuest in Figure 4.5a inherits its identity from both CurrentGuest and FutureGuest, but these entity sets, in turn, both inherit their identity from Guest. Thus, GuestNr becomes the one and only key attribute that identifies returning guests.
} 
we map the ISA hierarchy rooted at Activity in this way. The relational schema for Activity in Figure 4.5b is the same as it would be without the ISA hierarchy. HighCostActivity and FreeActivity, however, both inherit the primary key Description from Activity and include it as the primary key for their relational schemas along with all directly dependent attributes.

ER Mapping Rule \#6.2. Make a relational schema for only root entity sets. For this mapping, we collapse the entire ISA hierarchy to the entity set of the root generalization, ${ }^{3}$ so that all attributes of specializations become attributes of the root and all relationship sets associated with specializations become relationship sets associated with the root. We then map this single entity set to a relational schema in the usual way. After doing the mapping, we determine which attributes are nullable. All attributes that would not have been in the mapping if we had not collapsed the ISA hierarchy are nullable. In our relational schemas, we mark nullable attributes with a question mark. When we transform generic relational schemas to SQL create statements for implementation, we allow nullable attributes to have the value NULL; nonnullable attributes may not have the value NULL. In Figure 4.5 we map the ISA hierarchy rooted at Guest in this way. When collapsing the ISA hierarchy, the attributes of the three specializations, all become nullable attributes of Guest. As Figure 4.5b shows, these five attributes all have an appended question mark.

We might wonder if this mapping causes us to lose track of which guests are current guests, which are future guests, and which are returning guests. For this example we do not lose the information. According to the semantics of the ER model instance in Figure 4.5a only returning guests will have Discount values whereas current and future guests who are not returning guests will not have Discount values. Future guests will have a value for $\mathrm{NrOf}$ Reservations whereas current guests will not. Similarly, current guests will have extra charges, credit card numbers, and credit card expirations whereas future guests will not. Sometimes, however, it is not possible to know the specialization categories based on attribute values, and even when it is possible, we may wish to have a way to record the specialization categories. The following two additions to Mapping Rule \#6.2 show us how we can provide attributes in which we can record this information about specializations.

- ER Mapping Rule \#6.2a. Add a new attribute for each specialization. When mapping the generalization entity set to a relational schema, generate an additional attribute for every specialization entity set. Values for these attributes are Boolean, saying for each record of the relational schema whether the entity the record represents is or is not in the specialization. If we were to add these additional attributes for Guest, the relational schema for Guest in Figure 4.5b would instead be:

Guest(GuestNr, Name, ExtraCharges?, CreditCardNr?,

3 Although rare, if there are multiple roots, we collapse the hierarchy to all roots. Any entity set that is the specialization of multiple roots collapses to all of them. 
CreditCardExpiration?, NrOfReservations?, Discount?,

CurrentGuest, FutureGuest, ReturningGuest)

If we wish, we could omit ReturningGuest and just compute its value for a record as yes if both values for CurrentGuest and FutureGuest are yes and as no otherwise.

- ER Mapping Rule \#6.2b. Add only one new attribute representing all specializations. This mapping only applies when the specializations are mutually exclusive. If so, when mapping the generalization entity set to a relational schema, we only need to generate one additional attribute to represent all specializations. The specialization entity-set names can be the values for this new attribute. In Figure 4.5a, Room has three mutually exclusive specializations that designate the room type. We therefore generate a new attribute, RoomType, for the generalization entity set Room. The values for this attribute can be the names of the specialization entity set. The generated relational schema for Room in Figure 4.5b has the attributes Room Nr and RoomType. Values for RoomType would be "Single", "Double", and "Suite" or any other designating value to say that the room is a single room, a double room, or a suite.

ER Mapping Rule \#6.3. Make a relational schema for only the leaves in the hierarchy. The leaf entity sets inherit all attributes and all relationship sets from parents along a path all the way back to the root. ${ }^{3}$ This mapping only applies when union constraints are present all along all paths. If a union constraint were missing, there could be members of the entity sets in the hierarchy that would not appear in the leaf entity sets and thus would be lost in the implementation. Further, we usually only apply this mapping when mutual-exclusion is also present along all paths. If not, then members of the entity sets could appear in more than one leaf entity set and thus would appear as duplicates in the implementation, once for each leaf entity set in which a member appears. As an example, assume that there are only high-cost activities and free activities and thus that the constraint for the ISA hierarchy rooted at Activity in Figure 4.5a is a partition $(\uplus)$ constraint rather than a mutual-exclusion $(+)$ constraint. Applying the mapping in which we only represent the leaves of the ISA hierarchy, we would replace the three relational schemas Activity, HighCostActivity, and FreeActivity in Figure 4.5b by the following two relational schemas:

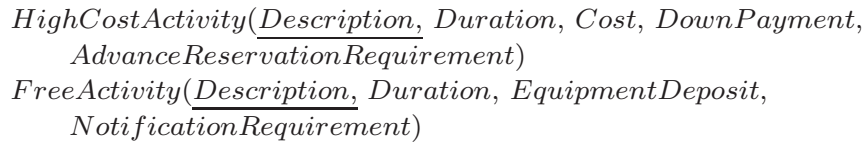

Observe that both HighCostActivity and FreeActivity include the attribute Duration as well as Description and that the connection to the IsSignedUpFor relational schema is accounted for through the Description

${ }^{3}$ If there are multiple roots, the leaves inherit from all roots. 
attributes. When we make a relational schema for only the leaves in an ISA hierarchy, we must account for all attributes and relationship-set connections of all ancestors of each leaf entity set.

General Principle \#4. Map ISA hierarchies to relational schemas by choosing to make a relational schema for (1) all entity sets in an ISA hierarchy, (2) only root entity sets, or (3) only leaf entity sets. Although there are guidelines that typically indicate which of the three mappings to use, making the best choice is often application dependent. Deciding among the possibilities depends on the ISA constraints and the number of attributes involved. Designers use the following rule-of-thumb guidelines.

- Select (1) when the generalizations and specializations all have many attributes.

- Select (2) when the specializations collectively have few attributes.

- When the specializations have no attributes, select (2a) either for an ISA union constraint or for an ISA with no constraint.

- When the specializations have no attributes, select (2b) either for an ISA partition constraint or for an ISA mutual-exclusion constraint.

- Select (3) for an ISA partition constraint, especially when there are many attributes for the specializations and few for the generalizations.

Often there is no obvious best choice. In this case the developer must choose one. Furthermore, in some complex cases, especially with large hierarchies, it may be best to make a separate choice for each individual ISA configuration in the hierarchy. In the end the mappings must account for representing all possible entities in every entity set in the ISA hierarchy and all possible relationships and attributes of these entities.

\subsubsection{Mappings for Complex Attributes}

Extended ER models allow for several types of complex attributes. Figure 4.6a includes examples for each type.

- A multi-valued attribute is an attribute whose values are sets of values. In an extended ER diagram, we denote a multi-valued attribute by a double oval. In Figure 4.6, ActivityInterest is a multi-valued attribute. Guests may be interested in several activities - one guest may be interested in the set of activities \{golf, horseback riding, canoeing\} while another guest may be interested in the set of activities \{chess, hiking, canoeing\}. View is also a multi-valued attribute, whose sets might be $\{$ Ocean, CityOverlook\} or just $\{$ Ocean $\}$ depending on what can be seen by looking out the window(s) of a room in the bed and breakfast establishment.

- A compound attribute is an attribute with component parts each of which is also an attribute. In an extended ER diagram, we denote compound attributes by attaching component attributes directly to the compound at- 


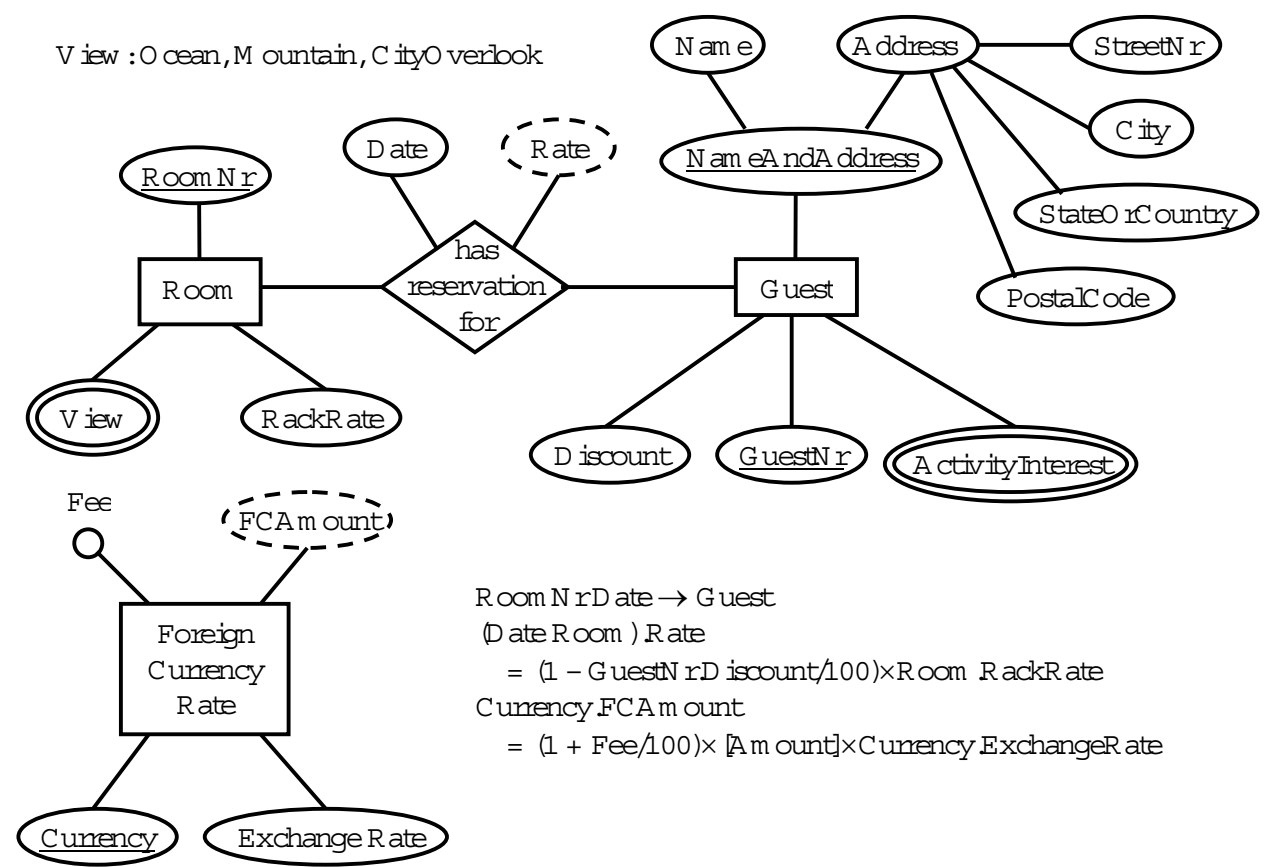

(a) ER Diagram.

Guest(GuestNr, Name, StreetNr, City, StateOrCountry, PostalCode, Discount)

GuestActivityInterest(GuestNr, ActivityInterest)

Room(RoomNr, RackRate, Ocean, Mountain, CityOverlook)

HasReservationFor(RoomNr, Date, GuestNr, Rate)

ForeignCurrencyRate(Currency, ExchangeRate)

Fee(Fee)

(b) Generated Schemas.

Fig. 4.6 Mappings for Complex Attributes: ER Diagram and Generated Schemas.

tribute. In Figure 4.6a, NameAndAddress is a compound attribute whose component attributes are Name and Address. The component attribute Address is also compound; its component attributes are StreetNr, City, StateOrCountry, and PostalCode.

- A computed attribute is an attribute whose value can be computed. In an extended ER diagram, we denote a computed attribute by a dashed oval. In Figure 4.6a, FC Amount and Rate are computed attributes.

- An entity-set attribute, called in other contexts a class attribute, is an attribute whose value is the same for all entities in the entity set and thus can be thought of as applying to the entire set of entities rather than each individual entity in the set. In an extended ER diagram, we denote an entity-set attribute by a small circle. In Figure 4.6a, Fee is an example. 
It is a percentage and is meant to be the fee collected by the bed and breakfast establishment for accepting payment in a foreign currency.

Figure 4.6b shows how we map the various complex attributes in Figure $4.6 \mathrm{a}$ to relational schemas. Basically, the mappings are straightforward applications of the general principles. The cardinality constraints for multivalued attributes make them have the properties of many-many relationship sets. The collapsing of compound-attribute trees make the leaves of these trees have the properties of directly dependent attributes. Computed attributes are the same as regular attributes except we may not need to store them. Although entity-set attributes could be treated as regular attributes, their singleton property makes them amenable to a different kind of mapping, making entity-set attributes an exception to the general principles.

ER Mapping Rule \#7. Fundamentally, multi-valued attributes are in a many-many relationship with the entity set to which they are connected. Each entity in an entity set $E$ with a multi-valued attribute $A$ relates to $n$ values $v_{1}, \ldots, v_{n}$ of $A$, and each value of $A$ relates to $m$ entities $e_{1}, \ldots, e_{m}$ in $E$. Thus, unless the number of values in $A,|A|$, is fixed and small, we treat $A$ as if it were another entity set $E^{\prime}$ in a many-many relationship with $E$; $E^{\prime}$ 's only attribute, and therefore its primary-key attribute, is $A$. When $|A|$ is fixed and small, it is possible to treat it as $|A|$ attributes $v_{1}, \ldots, v_{|A|}$ of $E$, whose values are Boolean stating whether an entity $e$ relates to that value or does not relate to that value.

- ER Mapping Rule \#7a. If entity set $E$ has a multi-valued attribute A, then if $P$ is the primary key of $E$, generate the relational schema $N(P, A)$. If the primary key of $E$ happens to be composite, $P$ represents the attribute list for the composite primary key. $N$ is a chosen name-often a concatenation of the name of $E$ and the name of $A$. The relational schema GuestActivityInterest in Figure 4.6b is an example. A guest can be interested in may different activities, and an activity can be of interest to many different guests. Thus, since Guest $N r$ is the primary key of Guest, we generate Guest $N r$ and ActivityInterest as the attributes and as the composite key for the relational schema.

- ER Mapping Rule \#7b. As an exception to ER Mapping Rule \#"7a, if entity set $E$ has a multi-valued attribute $A, n$ is the size of $A$, and $n$ is fixed and small, then if $A=\left\{V_{1}, \ldots, V_{n}\right\}$, add $V_{1}, \ldots, V_{n}$ as Boolean attributes to the relational schema formed for $E$. The relational schema Room in Figure $4.6 \mathrm{~b}$ is an example. As specified in a note in the diagram, a room can have only up to three views (Ocean, Mountain, or CityOverlook). Thus, for the multi-valued attribute View of Room, we add these three view names, Ocean, Mountain, and City as attributes to the relational schema. Values for these attributes are Boolean: if a front corner room has all three views, all three attribute values would have the value yes, and if a back center room looks out only on the mountains, the Mountain value would be yes and the Ocean and cityOverlook values would be no. 
ER Mapping Rule \#8. Treat each leaf attribute of a compound attribute tree $T$ of an entity set $E$ as an attribute of $E$; then map $E$ in the usual way. In addition, if any non-leaf node $N$ of $T$ is a key for $E$, form a composite key from the leaf attributes of the subtree rooted at $N$. In Figure 4.6a Guest has a compound attribute NameAndAddress. Its leaf attributes are Name, StreetNr, City, StateOrCountry, and PostalCode. Thus, for Guest we form a relational schema with these attributes along with the regular attributes Guest Nr and Discount. (Being multi-valued, ActivityInterest is not a regular attribute and is not included - neither are the non-leaf attributes of the compound attribute tree, NameAndAddress and Address.) Further, since the non-leaf attribute NameAndAddress is a key for Guest, we form a composite key from all its leaf attributes as Figure 4.6b shows.

ER Mapping Rule \#9. If a computed attribute is to be stored in the database, treat it as a regular attribute for the purpose of mapping it to a relational schema. When values for attributes are computed, we may or may not want to store their values in the database. If computed values serve to initialize a field and the field value may later change, we store the values. In this case, there must be an attribute for it in the generated relational schema. On the other hand, if the value is computed from other existing values whenever we need it, we need not store it. In this case, we ignore it when generating relational schemas. In our example in Figure 4.6a, Rate is an initial value, which depends on a guest's discount and the room's rack rate but which can be set to another value. Thus, we generate an attribute for Rate in the relational schema for HasReservationFor, the place it would go if it were a regular attribute. FCAmount, however, is only computed when a guest wants to know how much to pay if the amount owed is to be paid in a foreign currency. Thus, we do not generate an attribute for FCAmount in any relational schema.

ER Mapping Rule \#10. For an entity-set attribute $A$, we either ignore it or map it to a single-attribute, single-valued relation $A(A)$. Values for entity-set attributes may be constants established in the program code, may be values accepted as input values when needed, or may be stored in the database and updated occasionally. In our example, we store the fee value as a percentage number in the database and thus need the relational schema $\mathrm{Fee} \underline{(\mathrm{Fee})}$ as Figure 4.6b shows.

\subsubsection{Mappings for Mandatory/Optional Participation}

Figure 4.7a illustrates mandatory and optional participation in an ER diagram. We designate optional participation by placing a small "o" near a connection for attributes and relationship sets, and we designate mandatory participation by the absence of an "o." Optional participation for an attribute $A$ means that an entity in an entity set need not have a value for $A$; manda- 


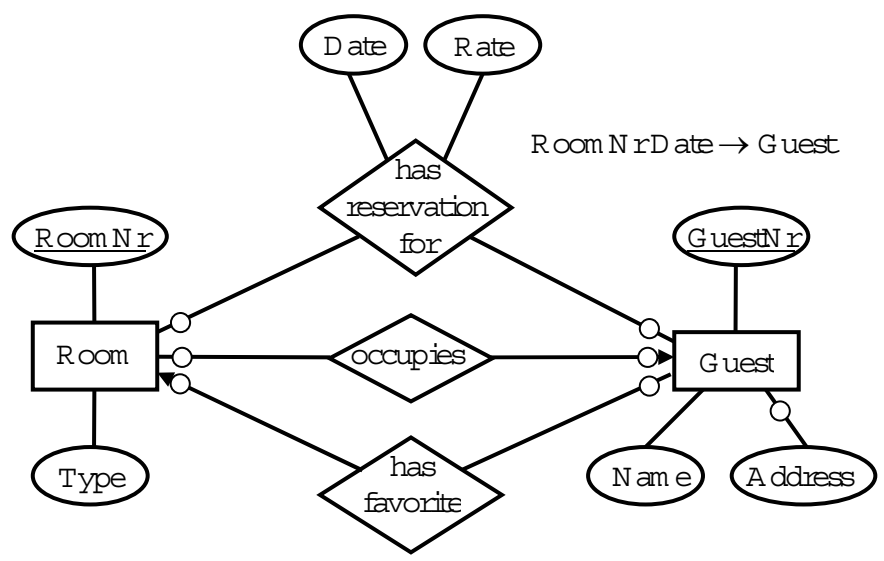

(a) ER Diagram.

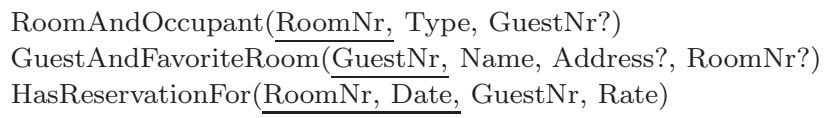

(b) Generated Schemas.

Fig. 4.7 Mappings for Mandatory/Optional Constructs: ER Diagram and Generated Schemas.

tory participation means that an entity must have a value. In Figure 4.7a a Guest need not provide an Address when registering (i.e., the database system will allow the Address field in a record for a Guest to be null). Optional participation for a relationship set $R$ means that an entity in an entity set need not participate in the relationship set; mandatory participation means that it must participate. For example, a Room in Figure 4.7a need not be occupied, need not be anyone's favorite, and need not be reserved by anyone. Similarly, someone recorded as a Guest in the database need not have a reservation, need not occupy a room, and need not have a favorite room. The database would allow, for example, a record to be kept for someone who had been a guest, but is not currently occupying a room, has no reservation, and has no particular favorite room.

Figure $4.7 \mathrm{~b}$ shows how we consider optionality when we map to relational schemas. As before, the question mark means that an attribute is nullable. When attributes are optional, they are nullable; when they are mandatory, they are not nullable. Thus, for example, Address has an appended question mark in GuestAndFavoriteRoom whereas Name does not. When attributes of a relationship set plus the primary-key attributes of the associated entity sets are mapped into a relational schema for an entity set, if participation in the relationship set is optional, these imported attributes are all nullable. When participation is mandatory, these attributes are not nullable. Thus, for example, Guest $N r$ is nullable in Room AndOccupant. Be- 
cause occupies is many-one from Room to Guest, the primary key for Guest, which is Guest $N r$, becomes one of the attributes of RoomAndOccupant, and because participation of a Room in the occupies relationship set is optional, Guest $N r$ is imported as a nullable attribute. Similarly, Room Nr is nullable in GuestAndFavoriteRoom since the has favorite relationship set is manyone from Guest to Room and Guest optionally participates in has favorite. Observe that the relationship set has reservation for is many-many and is not imported into either Room or Guest. Thus, there is no special mapping based on the optionality of reservations for rooms and guests.

ER Mapping Rule \#11. Mark all nullable attributes in generated relational schemas. Attributes that are the primary key of a relational schema or that are in the composite primary key of a relational schema are never nullable. All other attributes may be nullable and should be so marked if their value for a record can be NULL.

This rule is a little different from all other rules. It is written with respect to generated schemas. Thus, it does not say how to generate schemas, neither does it say exactly how to decide which attributes are nullable. Rather, it says which attributes are and are not potentially nullable, and it says that among those that are potentially nullable the database designer should decide which ones should allow null values. The reason for writing the rule this way is twofold:

1. Many ER diagrams never specify mandatory/optional participation (sometimes because the notation does not allow it, and sometimes just because it is not commonly done). Thus the nullable properties of attributes in relational schemas are often not derivable from the ER diagram.

2. When mandatory/optional participation can be specified in an ER diagram, even if someone specifies that an attribute that turns out to be part of the primary key of some relational schema should be nullable, it cannot be nullable. Relational database systems do not allow nulls in primary keys.

To illustrate Reason \#1, we can consider the ER diagrams in Figures 4.14.6 in which no optional participation explicitly appears. One view we can take for all these diagrams is that there is no optional participation. In this case, all generated relational schemas remain as they are. This point of view, however, does say something about the semantics of the schemas. For example, in the Room schema in Figure 4.1b, we can only record room numbers, type, and rate for occupied rooms. If we want to store this information for unoccupied rooms, we would be forced to enter some bogus Guest $N r$ (e.g., -1) and some bogus value for ExtraCharges (e.g., 0). It may be better to simply allow these attributes to be nullable. The same is true for address of a guest in the Guest schema in Figure 4.1b. Even if the guest is in the process of moving and has no address to give, or if the guest refuses to give an address, something (e.g., "none") must be entered. 
To illustrate Reason \#2, consider what it would mean if $R o o m N r$ for RoomAndOccupant in Figure 4.7b were marked as optional and thus allowed to be nullable. This means that some rooms would have no identifier - no room number. Suppose we try to store information about several no-number double rooms that currently have no occupants. Even if the database would let us store the room number as null, we would have trouble since we could not distinguish the rooms from one another. We would not even know how many unoccupied double rooms we have. This motivates the rule: Attributes of primary keys in a relational schema may not be null. Note that this rule does not say that key attributes cannot be null - only that primary-key attributes cannot be null. Suppose, for example, that a guest can have a guarantee number (Guarantee $\mathrm{Nr}$ ) that uniquely identifies the guest in addition to a guest number (Guest $N r$ ). We could then add Guarantee $N r$ as a key attribute to the attributes already in GuestAndFavoriteRoom in Figure 4.7b and let it be nullable so that not all guests would have to have a guarantee number. Note also that this rule does not say that primary-key attributes imported into a relational schema cannot be null. Indeed, Guest $N r$ is nullable in RoomAndOccupant, where it is not the primary key, even though it is the primary key for GuestAndFavoriteRoom.

General Principle \#5. Make attributes nullable if they can have NULL as their value. Attributes of primary keys in a relational schema are not nullable.

\subsection{UML Mappings}

In this section, we explain how to generate relational schemas from UML class diagrams. We base our procedure on the general principles, and thus this explanation serves as an example of how to develop mapping rules to map any conceptual model to relational schemas. In general, we first need to understand the syntactic features of the conceptual model and determine which corresponding ER features or extended ER features they denote. We can then map them to relational schemas in the same way we map ER features to relational schemas.

We illustrate our UML mappings using the class diagram in Figure 4.8 as an example. We begin by pointing out several syntactic features of this class diagram and explain how they correspond to (extended) ER features. First, UML does not provide a graphical notation for specifying keys. ${ }^{4}$ UML does, however, provide an Object Constraint Language $(O C L)$, in which we can express FDs. Figure 4.9 shows an example of how to specify the FD name location $\rightarrow$ BedAndBreakfast. Thus, to derive keys of classes in a class di-

4 UML does not use underlines to denote keys for classes; rather it uses underlines to denote static attributes - attributes that belong to classes, not attributes applicable to instances of classes. 


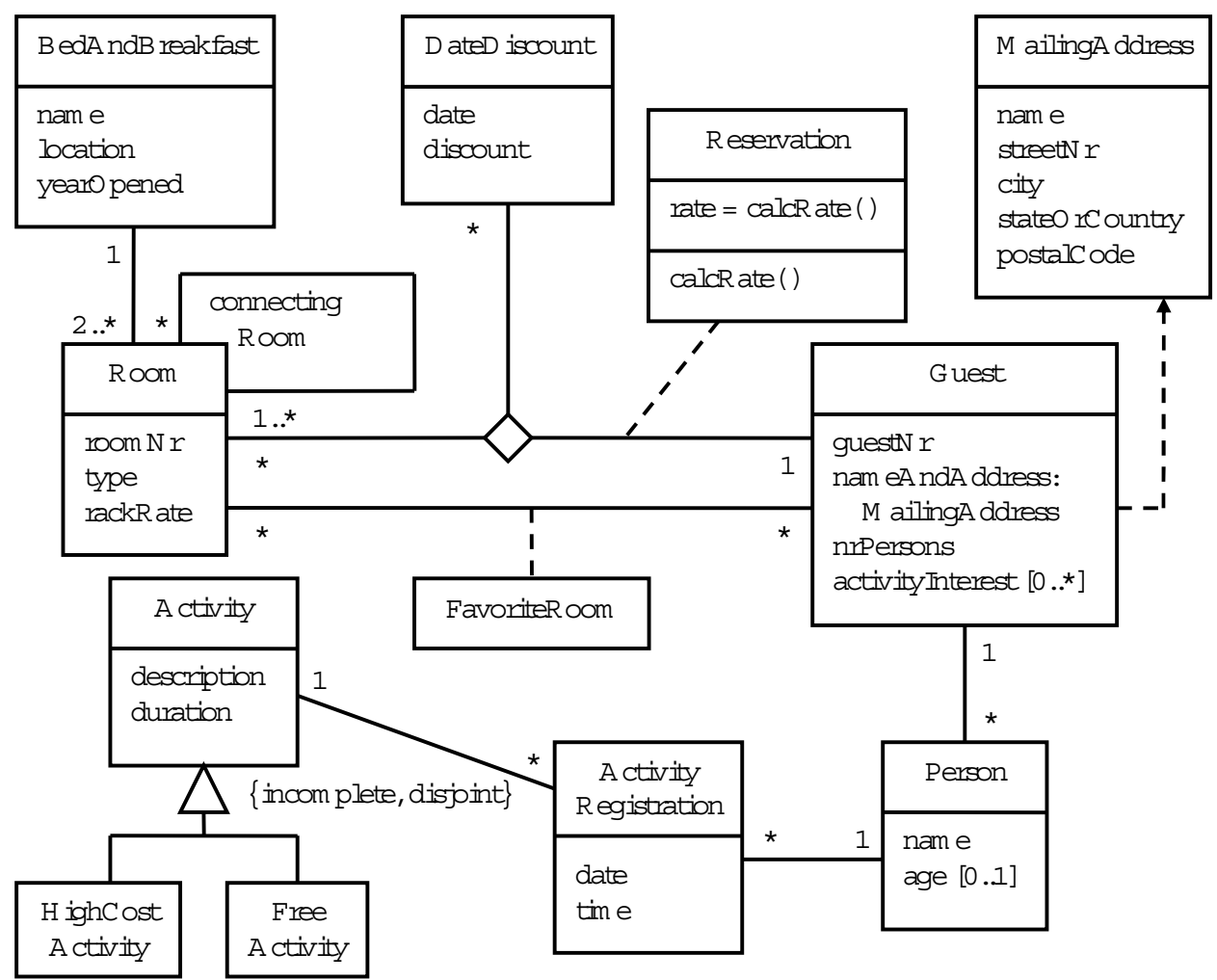

Fig. 4.8 UML Class Diagram

agram, we consult the OCL expressions associated with the class diagram. Second, we can use attribute multiplicity in class diagrams to specify optional attributes and multi-valued attributes. In Figure 4.8, age is an optional attribute and activityInterest is a multi-valued attribute. Third, UML allows the definition of a class to rely on the definition of another class. This allows us to specify a compound-attribute groups. For example, MailingAddress is a compound attribute group on which the class Guest depends. Fourth, UML allows operations to be defined in class diagrams. For example, rate, a computed attribute for the association Reservation, takes on the result of the operation calcRate() as its initial value.

Based on the general principles, we now present a high-level algorithm that generates relational schemas from a UML class diagram. As an example, we show how the UML diagram in Figure 4.8 along with the OCL constraints in Figure 4.9 map to the relational schemas in Figure 4.10.

Step 1 Based on General Principles \#1 and \#2, identify keys for each class. In our example, the UML OCL provides us with these keys. The left-hand- 


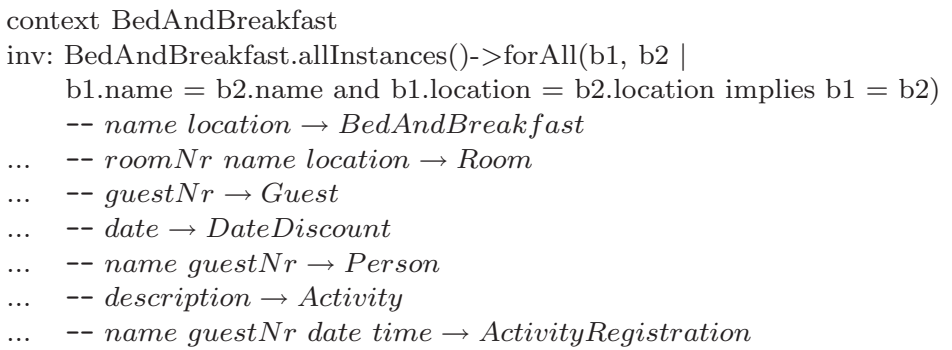

Fig. 4.9 OCL for an FD Plus Other FDs Declared for Classes in Figure 4.8.

BedAndBreakfast(name, location, yearOpened)

Room(roomNr, name, location, type, rackRate)

ConnectingRoom(roomNr, name, location, connectingRoomNr)

Guest(guestNr, name, streetNr, city, stateOrCountry, postalCode, nrPersons)

GuestActivityInterest(guestNr, activityInterest)

FavoriteRoom(roomNr, name, location, guestNr)

DateDiscount (date, discount)

Reservation(roomNr, name, location, date, guestNr, rate)

Person(name, guestNr, age?)

Activity(description, duration, costLevel?)

ActivityRegistration(name, guestNr, date, time, description)

Fig. 4.10 Schemas Generated from Figures 4.8 and 4.9.

side of each FD in Figure 4.9 is the key for the class on the right-hand-side of the FD.

Step 2 Based on General Principles \#1 and \#2, identify keys for each association. Usually multiplicity constraints of associations along with keys for classes determine these additional FDs. For example, if $A$ is an $n$-ary association that connects $n$ classes $C_{1}, \ldots, C_{n}$ whose primary keys are respectively $P_{1}, \ldots, P_{n}$ and the maximum value of the multiplicity for $C_{n}$ in this association is 1 , then the FD $P_{1} \ldots P_{n-1} \rightarrow P_{n}$ holds for $A$. In our example, we have room $N r$ name location date $\rightarrow$ Guest $N r$ for the ternary association Reservation in Figure 4.8. When an association has no max-1 multiplicity constraints, the key is the composite of the primary keys for all associated classes. For example, room $N r$ name location guest $N r$ is the key for FavoriteRoom.

Step 3 Based on General Principle \#4, determine how generalization/specialization should be mapped. Since the ISA constraints for the only generalization/specialization in Figure 4.8 are incomplete and disjoint and since there are no associations for the specializations, we choose to intro- 
duce one new attribute, costLevel representing all specializations as ER Mapping Rule \#6.2b suggests.

Step 4 Based on General Principle \#3, map classes to relational schemas. We generate a relational schema $R$ for a class $C$ as follows. $R$ has the following attributes:

- all the attributes of $C$ except (1) those whose multiplicity is greater than one (e.g., activityInterest in Figure 4.8) and (2) those compound attributes that reference another class (e.g., nameAndAddress in Figure 4.8);

- all leaf attributes of compound attributes (e.g., name, street Nr, city, stateOrCountry, and postalCode are all included in the relational schema for Guest);

- all attributes included in the primary key for $C$, if not already included (e.g., name and location from the class BedAndBreakfast are included in the relational schema for Room); and

- for each association $A$, if a key of $A$ is also a key of $R$, then (1) all attributes of $A$, if any, and (2) for each class $C^{\prime}$ connected to $C$ by $A$, the primary-key attributes of $C^{\prime}$ (e.g., description, the primary key of Activity, belongs in the relational schema for ActivityReservation).

Step 5 Based on General Principle \#3, map remaining associations to relational schemas. An association remains after Step 4 only if it is not one-one and not one-many or many-one. We generate a relational schema $R$ for each remaining association $A$ as follows. For each class $C$ connected by $A, R$ includes the primary-key attributes of $C$. FavoriteRoom and Reservation in Figure 4.10 are examples.

Step 6 Based on General Principle \#3, map multi-valued attributes to relational schemas. For each multi-valued attribute $M$ in a class $C$, generate a relational schema that includes $M$ and the primary-key attributes of $C$. The multi-valued attribute activityInterest in Guest in Figure 4.8 is an example, which yields the relational schema GuestActivityInterest in Figure 4.10.

Step 7 Based on General Principle \#5, identify nullable attributes. In our example age is nullable because it is a single-valued, optional attribute of Guest, and costLevel is nullable because the ISA hierarchy in Figure 4.8 has an incomplete constraint.

Once we have relational schemas for the database, like the ones in Figure 4.10, we can derive SQL DDL for a relational database. We illustrate here ${ }^{5}$ how to turn generated relational schemas into SQL table creation statements. Figure 4.11 shows our SQL DDL for the first three relational schemas in Figure 4.10. The translation is straightforward.

${ }^{5}$ We could have illustrated the derivation of SQL DDL for all earlier generated schemas as well as this one, but we only illustrate this derivation once. 
1. Obtain the name and basic attribute structure for the tables to be created directly from the generated schemas. As Figure 4.11 shows, the BedAndBreakfast table has the attributes name, location, and yearOpened. We can rename attributes to make them more understandable. BandBname and BandBlocation are preferable to name and location in Room (otherwise most people would read name in Room as the name of the room and location as the location of the room).

2. Represent the constraints captured in the diagram and generated schemas. The primary-key constraints come directly from the relational schemas, as do other uniqueness constraints. The foreign-key constraints come indirectly from the relational schemas. An attribute or attribute group that is not a key in a relational schema $R$ but is a key in a relational schema $S$ is a foreign key for $R$ that references $S$. BandBname and BandBlocation, for example, constitute a composite foreign key in Room that references the composite key name and location in BedAndBreakfast. As may be desirable, we also add check constraints, such as room $\mathrm{Nr} !=$ connectingRoom Nr. These constraints, however, are not derivable from the relational schemas we generate.

3. Add type declarations, which are usually only implicit in the conceptualmodel instance. The types varchar, number, and money are examples.

4. Reflect the null properties of the relational schemas in the SQL DDL. Primary-key attributes are not null by default. All other attributes are nullable unless otherwise specified by a not null constraint.

\subsection{Normal Form Guarantees}

When mapping conceptual models to database schemas, the question of normalization naturally arises. Are generated relational schemas fully normalized? By "fully normalized," we mean they are in PJNF — Project-Join Normal Form - which also implies that they are in 4NF, BCNF, 3NF, 2NF, and $1 \mathrm{NF}$. Interestingly, we can answer "yes" for conceptual-model diagrams that are canonical.

Although circular, the easiest way to define canonical is that when mapped according to the mapping rules or algorithms, every relational schema is fully normalized. In practice, many (if not most) diagrams are canonical. ${ }^{6}$ Giving a general statement that characterizes canonical for all types of conceptual models (or even for one type of conceptual model) is difficult especially if the characterization is to be given in the least constraining way. Many conceptual model instances, however, satisfy stronger than necessary conditions, and it

${ }^{6}$ Many argue that if conceptual-model diagrams are not canonical, they are not good quality diagrams. 


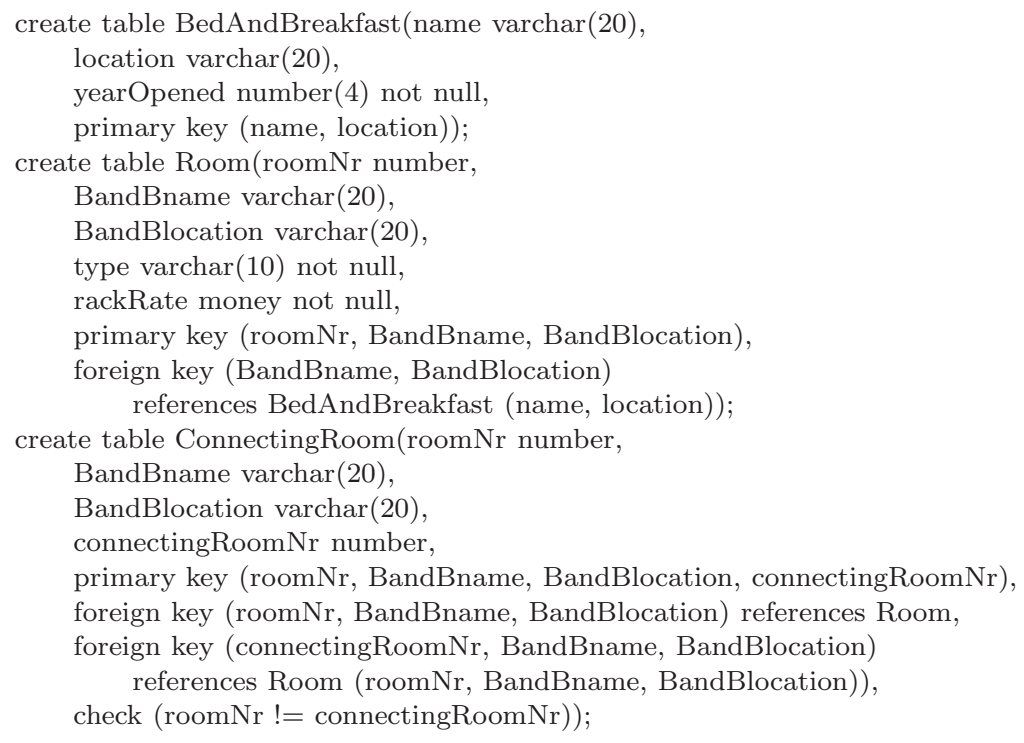

Fig. 4.11 SQL for Generated Schemas.

is easy to see that they are canonical. We can see, for example, that an ER model instance is canonical by checking the following criteria.

1. Each attribute is atomic (i.e., not decomposable into component attributes we wish to access in the database).

2. Each entity set has one or more keys (possibly inherited if the entity set is weak or in an ISA hierarchy), but has no other FDs among attributes with left-hand sides that are not keys.

3. Each many-many relationship set has one or more keys, but no other FDs among attributes with left-hand sides that are not keys.

4. Every $n$-ary relationship set $(n \geq 3)$ is fully reduced (i.e., we can't losslessly decompose it into two or more relationship sets).

5. There are no relationship-set cycles, or if there are cycles, then every path from one entity set to another is non-redundant in the sense that we cannot compute any relationship set as combinations of joins and projections of other relationship sets.

All the earlier ER diagrams in Sections 4.2 and 4.3 are canonical. Thus, all the generated relational schemas are fully normalized. Based on a similar set of criteria for UML, we can see that the UML diagram in Section 4.4 is also canonical and that its generated database schema is thus fully normalized.

To see that not all diagrams are canonical, consider Figure 4.12. This ER diagram violates the conditions listed above for being canonical. Supposing that we wish to access the first name and last name of the registered guest, 


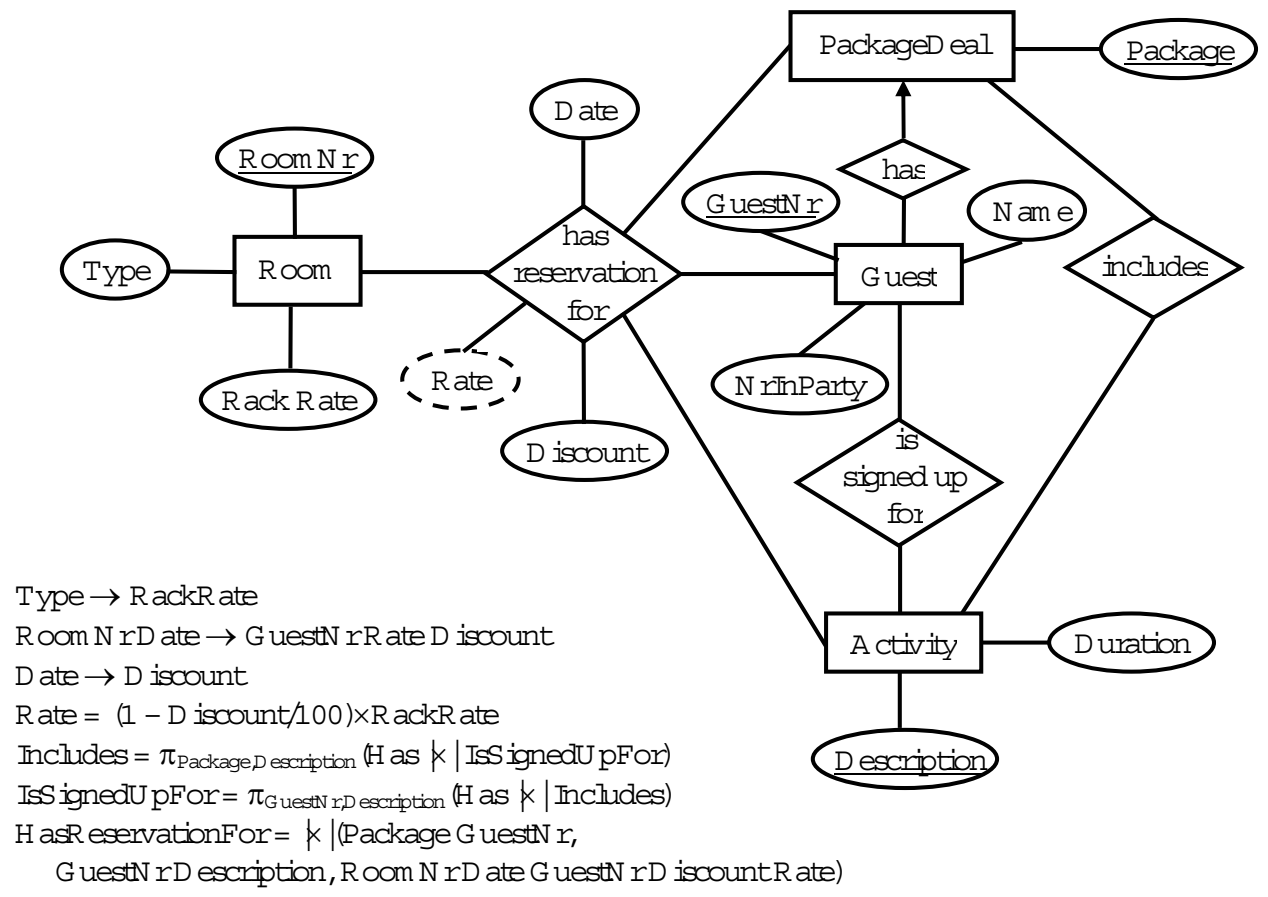

Fig. 4.12 ER Diagram with Constraints whose Standard Mapping will Yield Normal Form Violations.

Name is not atomic and thus is an example of a violation of Condition \#1. The diagram violates Condition \#2 because of the FD Type $\rightarrow$ RackRate, whose left-hand side is not a key for Room. The diagram violates Condition \#3 because the left-hand side of the FD Date $\rightarrow$ Discount is not a key for the relationship set has reservation for. (We are assuming for this example that the discount amount depends on the date - i.e., whether it is a weekend, weekday, holiday, during the off season, etc.) The diagram violates Condition \#4 because we can losslessly decompose has reservation for into three relationship sets: one between Package Deal and Guest (which also happens to be equivalent to the has relationship set), one between Guest and Activity (which also happens to be equivalent to the is signed up for relationship set), and one between Room and Guest. (Perhaps this original quaternary relationship set in Figure 4.12 arose because a designer was told that when guests make reservations they always also sign up for a package deal which includes certain activities.) The diagram violates Condition \#5 because of the cycle through the relationship sets has, includes, and is signed up for, in which includes and is signed up for are computable from the other two relationship sets in the cycle.

As we shall see in Section 4.5.1, if we use standard schema generation procedures, we would generate relational schemas that are not normalized. 
We should then normalize them using standard normalization techniques discussed in Chapter 6. We will also see in Section 4.5.2, however, that we can rearrange the diagram so that it has the same semantics but is canonical. If we then use standard schema generation procedures, the resulting relational schemas will be fully normalized. We will thus see that there are two approaches to ensure that generated relational schemas are fully normalized. (1) We can first generate relational schemas and then normalize using standard normalization techniques. (2) We can first canonicalize a conceptual-model diagram and then generate relational schemas.

\title{
4.5.1 Map - Then Normalize
}

\author{
Room(RoomNr, Type, RackRate)

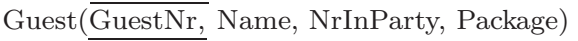 \\ Activity(Description, Duration) \\ HasReservationFor(RoomNr, Date, GuestNr, Rate, Discount, Package, Description) \\ IsSignedUpFor(GuestNr, Description) \\ Includes(Package, Description)
}

Fig. 4.13 Generated Relational Schemas - Not Normalized.

Figure 4.13 shows the relational schemas we would generate according to the standard ER mapping rules in Sections 4.2 and 4.3. We must now recognize the normal-form violations and fix them.

- Guest is not in $1 \mathrm{NF}$ because Name is not atomic. We replace Name by FirstName and LastName in Guest, which yields Guest(GuestNr, FirstName, LastName, NrInParty, Package).

- HasReservationFor is not in $2 \mathrm{NF}$ because of Date $\rightarrow$ Discount. We decompose HasReservationFor, which yields HasReservationFor (RoomNr,Date, GuestNr, Rate, Package, Description) and a new relational schema DateDiscount(Date, Discount).

- Room is not in 3NF because of Type $\rightarrow$ RackRate. We decompose Room, which yields Room(RoomNr, Type) and a new relational schema RoomType( Type, RackRate).

- The relationship set HasReservationFor is not in 4NF/PJNF because of the join dependency Package Guest Nr, GuestNr Description, Room Nr Date GuestNr Rate). (Note that Discount is missing because we have already decomposed HasReservationFor in a step above.) We thus decompose HasReservationFor according to the join dependency, which yields three relational schemas: HasReservationFor (RoomNr, Date, GuestNr, 
Rate), one whose schema is (Guest Nr,Description), and one whose schema is (GuestNr, Package). Since (GuestNr,Description) is the same as IsSignedUpFor, we discard it, keeping only IsSignedUpF (GuestNr, Package) is embedded in Guest, we discard it, keeping only Guest.

- Finally, we observe that the schema IsSignedUpFor is redundant because IsSignedupFor $=\pi_{\text {Guest } N \text {,Description }}($ Has $\bowtie$ Includes $)$. Note that although Includes is also redundant because Includes $=\pi_{\text {Package,Description }}($ Has $\bowtie$ IsSignedUpFor $)$, IsSignedUpFor and Includes mutually depend on each other. Thus, we can only remove one of them.

Normalizing the relational schemas as described results in the database schema in Figure 4.14.

\author{
Room( $\underline{\text { RoomNr}}$, Type) \\ RoomType(Type, RackRate) \\ Guest(GuestNr, FirstName, LastName, NrInParty, Package) \\ Activity(Description, Duration) \\ HasReservationFor(RoomNr, Date, GuestNr, Rate) \\ DateDiscount(Date, Discount) \\ Includes(Package, Description)
}

Fig. 4.14 Normalized Relation Schemas.

\title{
4.5.2 Normalize - Then Map
}

In the alternative approach to normalization, we first make the conceptualmodel diagram canonical. We then map it to relational schemas. Because the conceptual-model instance is canonical, the generated relational schemas will be normalized.

To make a conceptual-model instance canonical, we check for and ensure compliance with the basic criteria for characterizing canonical model instances presented earlier. We illustrate this process for ER model instances by making the diagram in Figure 4.12 canonical.

1. Non-atomic attributes. Assuming we wish to have FirstName and Last Name for Guest, Name is not atomic. We add these attributes, making Name a compound attribute as Figure 4.15 shows.

2. FDs whose left-hand sides are not keys. Recognizing the FD Type $\rightarrow$ RackRate as an FD whose left-hand side is not a key, we create a new entity set, RoomType, as Figure 4.15 shows. Type is a key attribute for RoomType, and RackRate is a regular attribute. Further, as Figure 4.15 


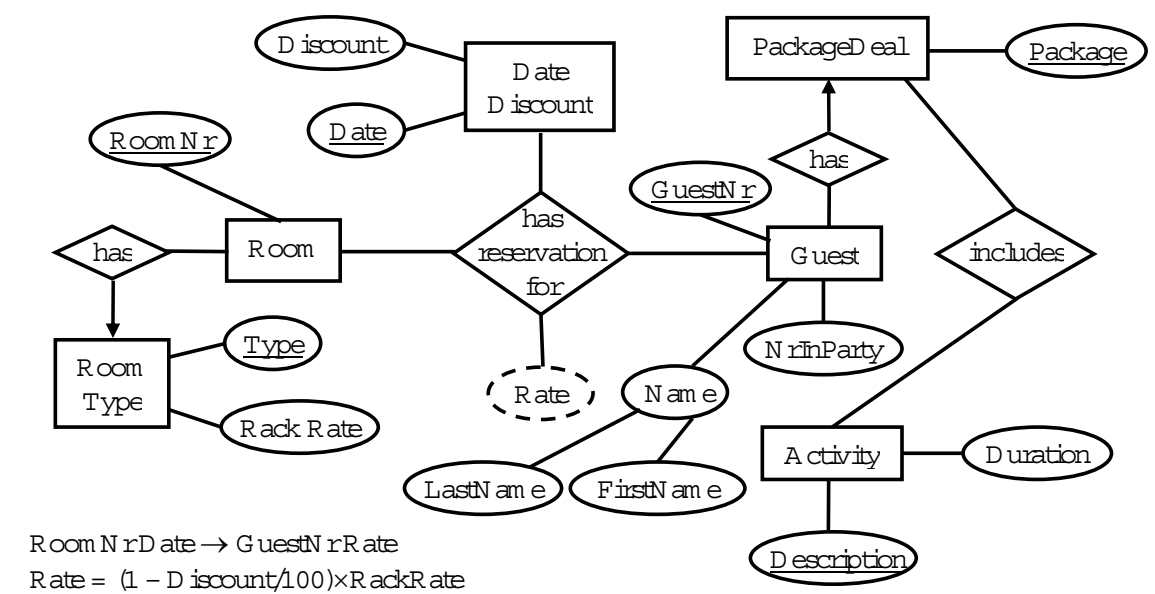

Fig. 4.15 ER Diagram Transformed to Generate Normalized Relation Schemas.

shows, because the FD Date $\rightarrow$ Discount is another FD whose left-hand side is not a key, we create another new entity set, DateDiscount. Its attributes are Date and Discount, with Date being a key attribute.

3. Reducible $n$-ary relationship sets. We can losslessly decompose the relationship set has reservation for. After adding the new entity set DateDiscount to this relationship set, the relationship set has reservation for has become a 5 -ary relationship set. We can decompose it losslessly into two binary relationship sets and one ternary relationship set. Since the two new binary relationship sets equate to the existing relationship sets has and is signed up for, we discard them. This leaves us with the ternary relationship set has reservation for in Figure 4.15.

4. Reducible cycles. The cycle of relationship sets from Guest to PackageDeal to Activity and back to Guest is a reducible cycle. We can remove either includes or is signed up for because either is computable from the other two relationship sets. We cannot remove both, however, because we need each one to compute the other. We choose to remove is signed up for as Figure 4.15 shows.

Figure 4.15 shows the resulting canonical ER diagram, and Figure 4.14 shows the relational schemas generated from the canonical ER diagram. It should not be a surprise that we obtain the same results whether we first generate relational schemas and then normalize or we first canonicalize the diagram and then generate relational schemas.

An alternative way to do conceptual-model-diagram canonicalization is to (1) transform the conceptual-model diagram to a hypergraph whose nodes are attributes and whose edges are relationship sets, ${ }^{7}(2)$ transform the hypergraph to a canonical hypergraph, and (3) map the canonical hypergraph

${ }^{7}$ Any ISA hierarchies remain intact without alteration. 
to relational schemas. There are several advantages of this approach: (1) Transforming a conceptual-model diagram to a hypergraph lets us explicitly add all connections among attributes. In particular, it lets us add the troublesome FD connections among attributes that are only implicit in diagrams that force attributes to be attached only to entity sets (e.g., ER) or classes (e.g., UML). ${ }^{2}$ (2) Since all the constraints needed for normalization appear directly in the diagram, the canonicalization process proceeds entirely with the diagram alone. (3) Finally, this approach leads to more straightforward mapping algorithms, and, as we shall see in Section 4.6, leads to mapping algorithms for object-based schemas and XML schemas.

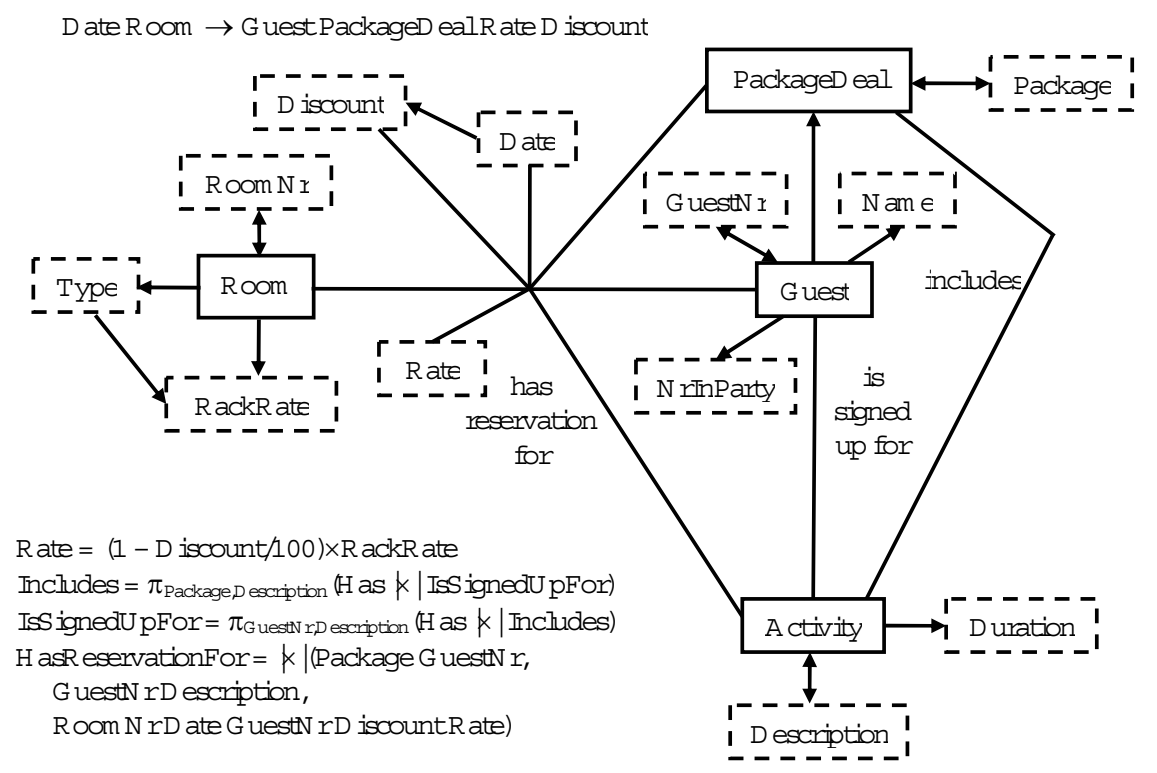

Fig. 4.16 Hypergraph Generated from the ER Diagram in Figure 4.12.

We illustrate this approach beginning with the non-canonical ER diagram in Figure 4.12, which we first convert to the non-canonical hypergraph in Figure 4.16. We convert an ER diagram to a hypergraph as follows.

1. Make every attribute be a lexical node in the hypergraph. Lexical refers to readable/writable data; lexical nodes represent the data we store in a database. We represent lexical nodes by dashed boxes as Figure 4.16 shows.

2 Some conceptual models (e.g., ORM [Hal95] and OSM [EKW92]) are directly based on hypergraphs and already include all connections among attributes. These conceptual models need no transformation to hypergraphs. For these hypergraph-based conceptual models, the canonicalization and mappings to relational schemas proceed as we describe here. 
2. Make every entity set be a non-lexical node in the hypergraph. Nonlexical refers to real-world objects that, if represented in a database, would be represented by object identifiers. We represent non-lexical nodes by solid boxes; Room, Guest, PackageDeal, and Activity are the non-lexical nodes in Figure 4.16.

3. Make every relationship set be an edge in the hypergraph. The relationship set has reservation for, for example, connects the four nonlexical nodes that were originally entity sets and the three lexical nodes that were originally attributes as Figure 4.16 shows. If there is a functional relationship among all the nodes of the relationship set, we represent this functional relationship set by marking the functionally determined nodes with arrowheads. The has relationship set between Guest and PackageDeal is an example. If there are other functional relationships among some or all the nodes, we add an edge for each one. To keep the diagram from becoming too cluttered, we may visually add these additional edges as FDs (but they are nevertheless edges in the hypergraph). Figure 4.16 includes the FD Date Room $\rightarrow$ Guest PackageDeal Rate Discount, which is a functional edge relating all nodes of the 7-ary relationship set except Activity.

4. Make every connection between an entity set and its attributes be an edge in the hypergraph. For multi-valued attributes, the edge is manymany (non-functional). For compound attributes, the edge is functional from entity set to leaf attribute, one edge for every leaf attribute. (Nonleaf attributes are discarded.) For all other attributes, the edge is functional from entity set to attribute. The functional edge from Activity to Duration in Figure 4.16 is an example. For singleton key attributes we also add a functional edge from attribute to entity. When we have a functional edge between an entity set and an attribute in both directions, we combine them as a single, bi-directional, one-one edge. In Figure 4.16, Room $\mathrm{Nr}$ for Room, GuestNr for Guest, Package for PackageDeal, and Description for Activity are all examples. For each composite key consisting of $n$ attribute nodes, we add an $(n+1)$-ary functional edge from the $n$ attribute nodes constituting the composite key to the entity-set node. We also combine the $(n+1)$-ary functional edge with the $n$ functional edges from the entity set to these attribute nodes to form a single, one-one edge between the entity-set node and the $n$ attribute nodes constituting the composite key.

5. For every FD among lexical nodes, add an edge. For our example, we add the edges Type $\rightarrow$ RackRate and Date $\rightarrow$ Discount as Figure 4.16 shows. 
We next make a non-canonical hypergraph canonical in three main steps. ${ }^{8}$ We illustrate these steps by converting the non-canonical hypergraph in Figure 4.16 to the canonical hypergraph in Figure 4.17.

1. Decompose all hyperedges that can be losslessly decomposed. In Figure 4.16 we decompose the 7 -ary edge along with the edge represented by the FD to a 4-ary functional edge Room Date $\rightarrow$ Guest Rate plus several other edges, all of which eventually turn out to be redundant. Figure 4.17 shows this 4-ary edge, but none of the redundant edges.

2. Remove all redundant edges and all redundant hyperedge components. In addition to the redundant edges just generated and ignored, we also remove the Room-RackRate edge and the Guest-Activity edge.

3. Merge each non-lexical node with its key (any one key, if there is more than one choice). For example, we merge Room with its primary key Room Nr, which, as Figure 4.16 shows, are in a one-one correspondence. The result of this merge is the lexical node Room $N r$, which has been merged with Room as Figure 4.17 shows. Similarly, we merge Package with PackageDeal, GuestNr with Guest, and Description with Activity.

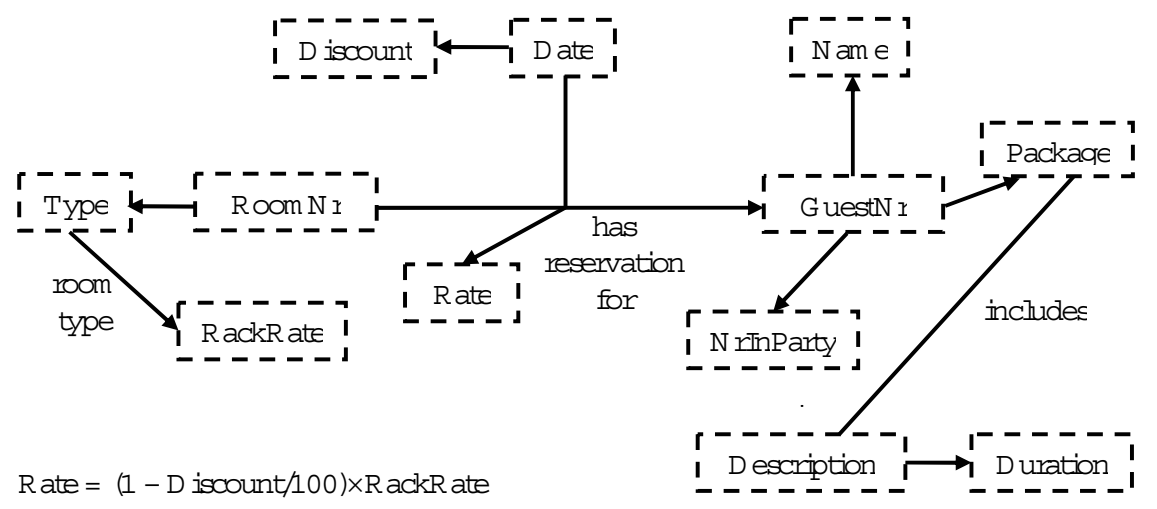

Fig. 4.17 Canonical Hypergraph.

\footnotetext{
${ }^{8}$ Usually these three main steps are enough. Exceptions arise (1) when the hypergraph is cyclic after redundancies have been removed and (2) when optional participation interferes with our ability to capture all element values. An example of the first would be an additional edge between Description and Duration where one means the average duration for an activity and the other means the maximum allowable duration. In this case, we need to qualify Duration to be, say, AveDuration and MaxDuration, and generate the relational schema for Activity with three attributes, Duration, AveDuration, and MaxDuration. An example of the second would be optional participation for Name where we might want to keep all names of guests even if they have no guest number. In this case, we need a separate table for Name alone since we cannot capture all names in the Guest relational schema, which demands a Guest Nr for every Guest. We can resolve both of these issues by adding roles. See [Emb98] for details.
} 
Once we have a canonical hypergraph, the conversion to a relational database schema is straightforward. With one mapping rule, we can obtain a set of normalized relational schemas:

Every edge becomes a relational schema. If the edge is functional in only one direction, the tail attribute(s) constitute the key. If the edge is functional in both directions (from tail(s) to head(s) and from head(s) to tail(s)), the head attribute(s) constitute a key as well as the tail attribute(s). If the edge is not functional, all the connected attributes constitute the key.

For example, Room Nr $\rightarrow$ Type is a functional edge whose generated relational schema is (Room Nr, Type), and RoomNr Date $\rightarrow$ Guest Nr Rate is another functional edge whose generated relational schema is (RoomNr, Date, GuestNr, Rate). The edge between Package and Description is a nonfunctional, and thus its generated relational schema is (Package, Description). We have no edge that is functional in both directions. To illustrate a bidirectional edge, suppose, as in Figure 4.1, that in addition to room numbers, rooms in a bed and breakfast establishment also have identifying names. We would then have another attribute Room Name in a one-to-one correspondence with $R o o m N r$. In this case, we would generate the relational schema (Room Nr, RoomName) in which both Room Nr is a key and RoomName is a key.

Although this single rule generates relational schemas that are all normalized, it fragments the database into more relational schemas than necessary. Thus, to finalize the relational schemas for the database, we merge as many as we can without violating any normal form using the following simple rule:

Merge schemas that have a common key.

For example, we would merge (Guest Nr, Name), (GuestNr, Package), and (GuestNr, NrInParty), because they all have the key Guest $N r$. We also add a name as we merge so that we have a standard relational schema. We would thus obtain Guest(GuestNr, Name, Package, NrInParty). Note that we would not merge ( $\underline{\text { RoomNr, T}} y p e)$ and (RoomNr, Date, Guest $N r$, Rate) because they do not have a common key - $\overline{R o o m N r}$ is not the same as the composite key Room Nr Date. Forming the relational schemas from Figure 4.17 and then merging those that have a common key results in the relational schemas in Figure 4.14, except that Name appears in place of FirstName and LastName since we started with Name rather than FirstName and Last Name in Figure 4.12.

Note that we have said nothing about ISA hierarchies. This is because there is no change to the way we map ISA hierarchies to relational schemas. There is a change, however, when we convert non-canonical hypergraphs to canonical hypergraphs. When we merge a non-lexical node with its key, we propagate this merge all the way down the hierarchy. Thus, for example, when we convert the ER diagram in Figure 4.5 to a canonical hypergraph, 
every node in the ISA hierarchy rooted at Guest becomes a lexical node with the name Guest Nr. ${ }^{9}$ If we choose to have a relational schema for every node in the ISA hierarchy, we simply do not merge relational schemas in the ISA hierarchy that share a common key. If we choose to have a relational schema only for the root of the ISA hierarchy, we merge in the usual way. If we choose to have relational schemas only for the leaves of the ISA hierarchy, we merge along every path from the root to each of the leaves.

\subsection{Mappings for Object-Based and XML Databases}

In this section, we show how to generate generic hierarchical structures, called scheme trees, from a canonical hypergraph. Since scheme trees are generic hierarchical structures, it is straightforward to map scheme trees to database schemas that support hierarchical data. As an example, we show how to map scheme trees to object-relational database schema instances and XML schema instances. Since object-oriented databases are similar to objectrelational databases, our example for object-relational databases serves for object-oriented databases as well.

The central idea of generating scheme trees is to ensure that each instance of a hyperedge in a canonical hypergraph only appears once in a schemetree instance. To do so, our scheme trees observe the many-one and one-one constraints in the hypergraph. We capture the essence of the idea in the following algorithm. ${ }^{2}$

While there is an unmarked hyperedge, do:

Select a subset $V$ of vertices in an unmarked hyperedge $E$

to be the root node of a new scheme tree $T$.

While we can add an unmarked hyperedge $E$ to $T$, do:

(We can add an unmarked edge $E$ if the following conditions hold: $E$ must have a nonempty intersection $S$ of vertices with $T$. A node $N$ must exist in $T$ such that the set $S$ is contained in the union of the nodes above or equal to $N$ and $S$ functionally determines the union of the nodes above or equal to $N$.)

Add $E$ to $T$ as follows:

If $S$ functionally determines $E-S$

Add the vertices in $E-S$ to the node $N$. Else

\footnotetext{
9 To keep the various nodes straight, we should add comments to the diagram. Except that these comments help us keep the nodes conceptually straight and may help us choose names for relational schemas, we can ignore these comments.

2 The algorithm is a simplified version of the heuristic $n$-ary algorithm in [ME06], which generates scheme trees from a canonical hypergraph.
} 
Make a node consisting of the vertices in $E-S$ and add it to the tree as a child node of $N$.

Mark $E$ as used.

If we select Package (a subset of the vertices in the edge Guest $N r \rightarrow$ Package) as the root node, this algorithm generates the scheme tree in Figure 4.18a. Having selected $\{$ Package $\}$ as the root for $T$ and $E=\{$ Package, Guest $N r\}$ as the edge we are considering, we see that all conditions of the while loop trivially hold (as they always do for the initial selection of a root and an edge). Since $S=\{$ Package $\}$ does not functionally determine $E-S$ $=\{G u e s t N r\}$ (it is actually the other way around), we add $\{$ Guest $N r\}$ as a child node of the node $\{$ Package $\}$. Continuing, we next consider the edge Guest $N r \rightarrow$ Name. Here, the conditions of the while loop also hold with $S$ $=\{G$ uest $N r\}, N=\{$ Guest $N r\}$, and Guest $N r \rightarrow$ Guest $N r$ Package. Since $S$ functionally determines $E-S=\{G u e s t N r$, Name $\}-\{$ Guest $N r\}=$ $\{N a m e\}$, we add Name to the node containing Guest $N r$. Similarly, we add NrInParty to this node, thus completing the second node in Figure 4.18a. We next consider the edge RoomNr Date $\rightarrow$ Guest Nr Rate. The intersection $S$ with $T$ is $\{G u e s t N r\}$, which is contained in the node $N$ we just created. Further, Guest Nr $\rightarrow$ GuestNr Name NrInParty Package so that it functionally determines the union of $N$ and the node above $N$ (the root). Since $S$ does not functionally determine $E-S$ (i.e., $\{$ Guest $N r\}$ does not functionally determine $\{$ Room Nr, Date, Rate $\}$ ), we make a new node consisting of $\{$ Room Nr, Date, Rate $\}$ and place it below $N$ as Figure 4.18a shows. Continuing, we next consider the nonfunctional edge \{Package, Description\}. The nonempty intersection $S$ with the scheme tree $T$ we have built so far is $\{$ Package $\}$. Since $\{$ Description $\}$ does not functionally determine $\{$ Package $\}$, we form a new node consisting only of $\{$ Description $\}$ and add it as another child node of $\{$ Package $\}$, resulting in the scheme tree $T$ in Figure 4.18a. Of the remaining four edges, Type $\rightarrow$ RackRate has an empty intersection with $T$, and the rest (Date $\rightarrow$ Discount, RoomNr $\rightarrow$ Type, and Description $\rightarrow$ Duration) do not satisfy the functional-dependency condition of the while loop for the scheme tree in Figure 4.18a.

Figure 4.18b shows the textual representation for the scheme tree in Figure 4.18a. In the textual representation, each node appears within parentheses with a star to indicate repetition; parenthesized nodes appear in line according to their nested position in the scheme tree.

Choosing the best starting place to run the algorithm depends on the semantics of the application. In our example, starting with Package is probably not best. The main point of the application is to rent rooms to guests, not about the activity packages they may choose. Thus, most of the processing is likely to be about looking up guests. Hence, a likely better starting place for our scheme-tree algorithm is to choose GuestNr as the root. When we start with Guest Nr as the root of the first tree and run the algorithm repeatedly until all edges are in some scheme tree, we obtain the scheme trees in Figure 4.19. 
(a) Tree Representation.

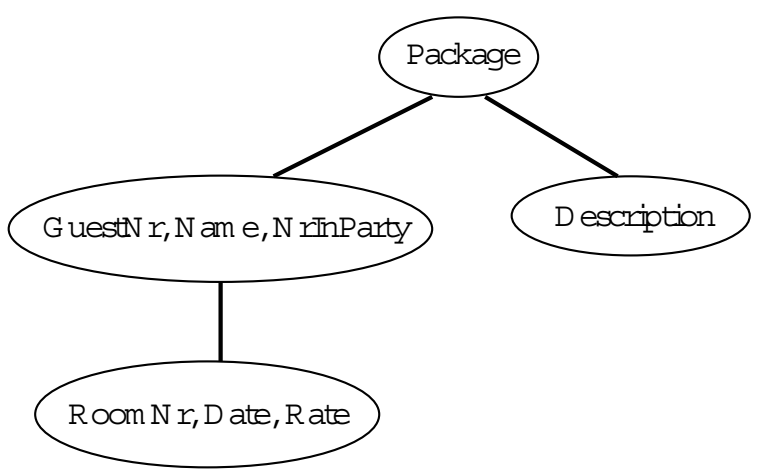

(Package, (GuestNr, Name, NrInParty, (RoomNr, Date, Rate)*)*, (Description)* $)^{*}$

(b) Textual Representation.

Fig. 4.18 Scheme-Tree Representations.

In Figure 4.19 we have marked keys in the usual way. In our example, Guest Nr, Date, Type, and Description values must all be unique in the usual way we expect key values to be unique. Since Room Nr Date $\rightarrow$ Guest $N r$, Room Nr-Date value pairs must be unique in the nested relation in which they appear. Similarly, since Room $N r \rightarrow$ Type, Room $N r$ values must also be unique in the nested relation in which they appear.

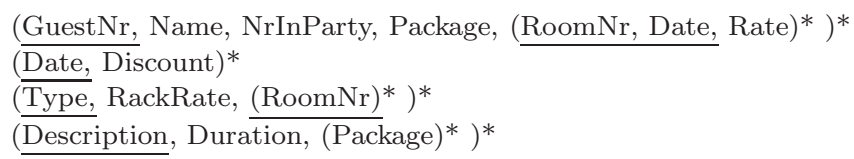

Fig. 4.19 Generated Scheme-Tree Forest.

Figure 4.20 shows an object-relational database schema instance derived from the first scheme tree in Figure 4.19. The main idea of this derivation is to scan a scheme tree bottom up and generate object types and collection types as we go up the scheme tree. Specifically, consider a leaf node $v$ whose parent is $u$. We generate an object type $t_{v}$ for $v$ such that $t_{v}$ has all the attributes in $v$. Then, we create a collection type of $t_{v}$. Afterwards, we generate an object type $t_{u}$ for $u$ such that $t_{u}$ has all the attributes in $u$ and a field whose type is the collection type of $t_{v}$. We then continue this process up to the root node of the scheme tree. Thus, for example, for the first scheme tree in Figure 4.19, we create an object type called reservation that includes three fields: room $N r$, reservationDate, and rate. We then create a collection type called collection OfReservation, which is a variable length array that stores 
reservation objects. Finally, we create an object type called guest for the root node. Note that there is a field called reservations in guest whose type is collection Of Reservation. To store guest objects, we create tableOfGuest a table of this type. Unfortunately, declared key constraints are not typically provided by object-relational databases. Thus, as for any constraint we wish to enforce that is not directly supported by the database, we must provide our own code to check and enforce it.

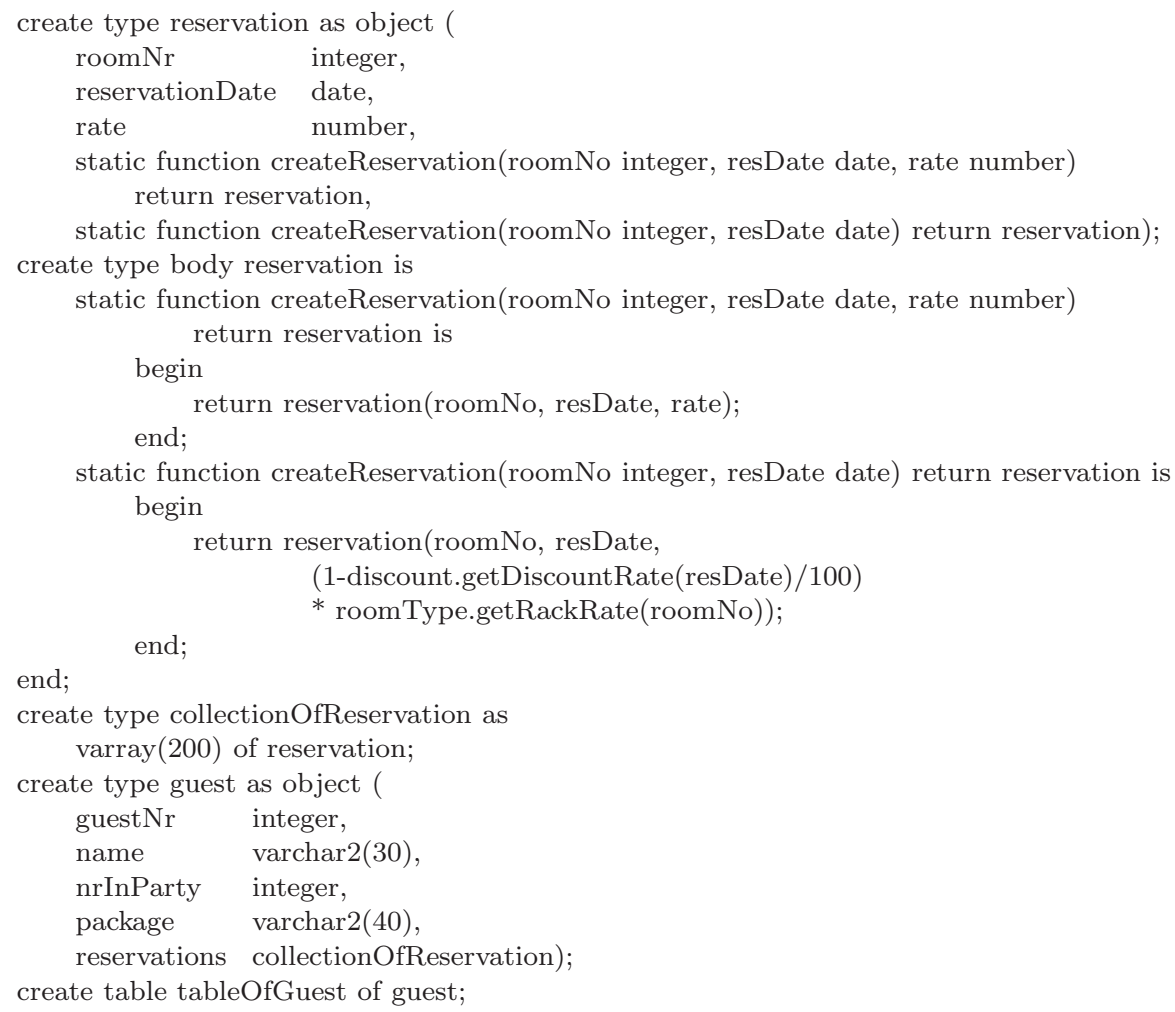

Fig. 4.20 Derived Object-Relational Database Schema Instance.

Figure 4.21 shows the first and last parts of a derived XML schema instance for the generated scheme-tree forest in Figure 4.19. Several derivations are possible; Figure 4.21 shows one derived as follows. For each nesting of a scheme tree we provide two names - one for describing the group and one describing an individual in the group. Thus, for the scheme tree (Type, RackRate, $(\text { RoomNr)* })^{*}$ we introduce the name RoomTypes for the group, RoomType for the individuals in the group, Rooms for the nested group, and Room for the individuals in the nested group. We nest these names ap- 
propriately as Figure 4.21 shows. We then put the attributes in their proper place - we nest Type and RackRate under RoomType and Room Nr under Room. The type for Type is xs:ID making it unique throughout the document. Since Type appears nowhere else in the scheme-tree forest in Figure 4.19, this simple declaration is sufficient. For Room Nr, which does appear in one other scheme tree, we scope the extent of uniqueness to be within RoomTypes and make a key declaration as Figure 4.21 shows. Further, when an attribute appears in more than one scheme tree, we use ref to reference a single declaration for its type. Room $N r$ is an example; its type declaration along with the type declarations for Date and Package which also appear in more than one scheme tree appear globally at the end of the XML schema instance in Figure 4.21. In the first part of the XML schema instance in Figure 4.21, we have declarations for the roots of each of the scheme trees, Guests, Activities, DateDiscounts, and RoomTypes all under the ultimate root, Document. We allow each of them to be optional except RoomTypes. (Although there may be no guests, activities, or date-discounts, if there are no rooms, there is no bed and breakfast establishment.)

\subsection{Additional Readings}

From the beginning, mappings from conceptual-model instances to database schemas have been an important part of the conceptual-modeling literature. Peter Chen's seminal article on the ER model includes an extensive discussion of mapping the ER model to various database models [Che76]. Ever since the appearance of this seminal article, discussions of mapping conceptual-model instances to database schemas have continued. Notable, along the way, are an ACM Computing Surveys article [TYF86] and a foundational book on conceptual database design [BCN92]. Most current database text books (e.g., [UW02, EN04, SKS06]) contain chapters on mapping conceptual models to database schemas.

Normalization concerns have also always been a part of mapping conceptualmodel instances to database schemas. Chen's seminal article [Che76] addressed normalization, and his mappings did yield relations in $3 \mathrm{NF}$ under his assumptions. Along the way other researchers have added insights about normalization. Researchers have suggested both the map-then-normalize approach [TYF86] and the normalize-then-map approach [CNC81, Lin85]. In

[BCN92] the authors take the point of view that ER diagrams are not of good quality unless they are canonical, and they talk about canonicalizing ER diagrams as one way to help create high-quality diagrams. The hypergraph approach to normalization appeared initially in [EL89]; full details appeared later in [Emb98].

Only recently have articles appeared that describe the process of mapping conceptual models for object-based, object-oriented, and XML databases. 


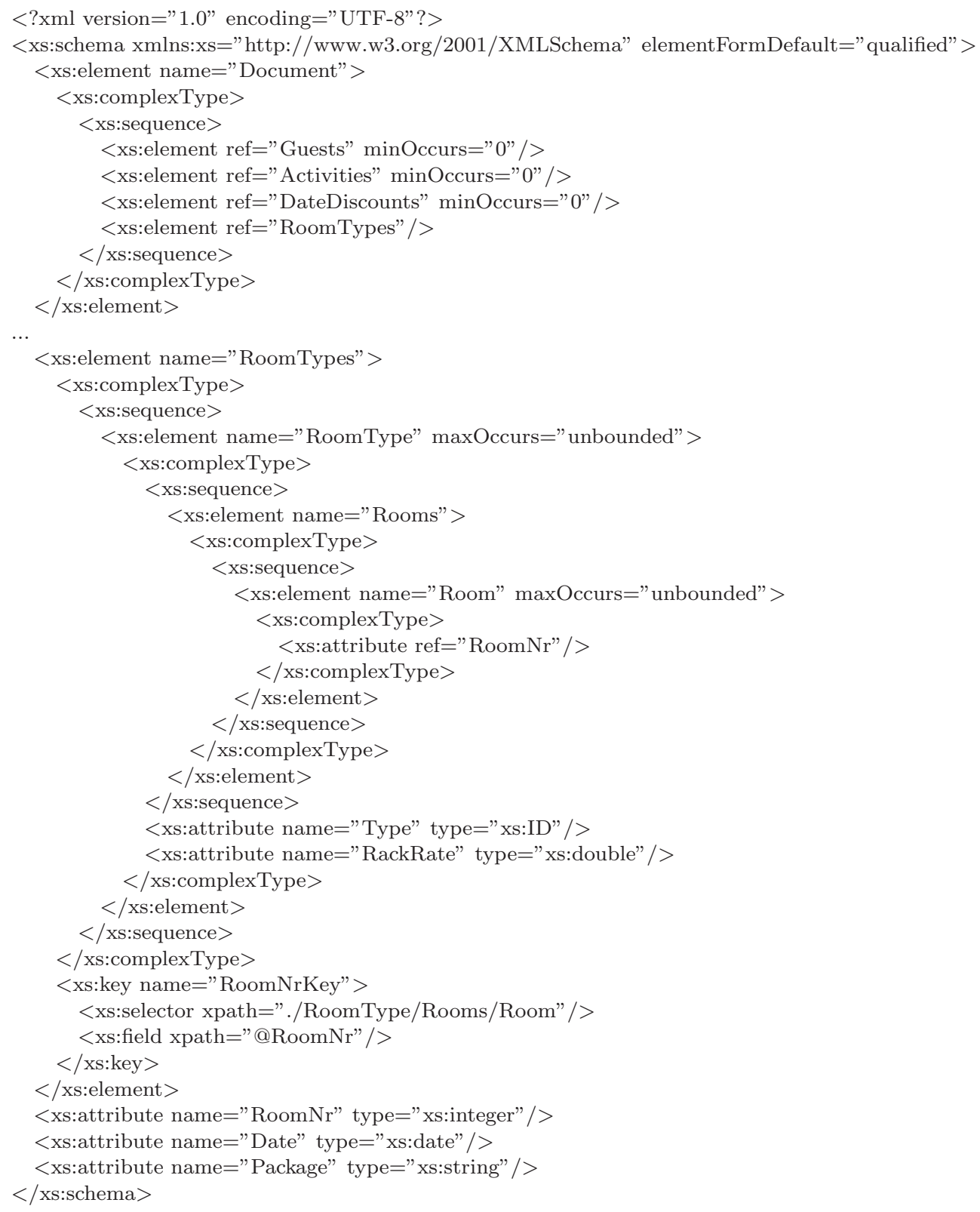

Fig. 4.21 Derived XML Schema Instance. 
Some initial thoughts appeared in [Emb98, EM01]. Proofs that generated scheme trees have no redundancy appeared later [ME06].

\section{References}

[BCN92] C. Batini, S. Ceri, and S.B. Navathe. Conceptual Database Design: An EntityRelationship Approach. The Benjamin/Cummings Publishing Company, Inc., Redwood City, California, 1992.

[Che76] P.P. Chen. The entity-relationship model — toward a unified view of data. ACM Transactions on Database Systems, 1(1):9-36, March 1976.

[CNC81] I. Chung, F. Nakamura, and P.P. Chen. A decomposition of relations using the entity-relationship approach. In Proceedings of the 2nd International Conference on Entity-Relationship Approach to Information Modeling and Analysis (ER'81), pages 149-171, Washington D.C., October 1981.

[EKW92] D.W. Embley, B.D. Kurtz, and S.N. Woodfield. Object-oriented Systems Analysis: A Model-Driven Approach. Prentice Hall, Englewood Cliffs, New Jersey, 1992.

[EL89] D.W. Embley and T.W. Ling. Synergistic database design with an extended entity-relationship model. In Proceedings of the 8th International Conference on Entity-Relationship Approach (ER'89), pages 118-135, Toronto, Canada, October 1989 .

[EM01] D.W. Embley and W.Y. Mok. Developing XML documents with guaranteed 'good' properties. In Proceedings of the 20th International Conference on Conceptual Modeling (ER2001), pages 426-441, Yokohama, Japan, November 2001.

[Emb98] D.W. Embley. Object Database Development: Concepts and Principles. AddisonWesley, Reading, Massachusetts, 1998.

[EN04] R. Elmasri and S.B. Navathe. Fundamentals of Database Systems. AddisonWesley, Boston, Massachusetts, fourth edition edition, 2004.

[Hal95] T. Halpin. Conceptual Schema \&s Relational Database Design. Prentice Hall of Australia Pty. Ltd., Sydney, Australia, second edition, 1995.

[Lin85] T.W. Ling. A normal form for entity-relationship diagrams. In Proceedings of the 4th International Conference on Entity-Relationship Approach (ER'85), pages 24-35, Chicago, Illinois, October 1985.

[ME06] W.Y. Mok and D.W. Embley. Generating compact redundancy-free XML documents from concptual-model hypergraphs. IEEE Transactions on Knowledge and Data Engineering, 18(8):1082-1096, August 2006.

[SKS06] A. Silberschatz, H.F. Korth, and S. Sudarshan. Database System Concepts. McGraw-Hill, New York, New York, fifth edition, 2006.

[TYF86] T.J. Teorey, D. Yang, and J.P. Fry. A logical design methodology for relational databases using the extended entity-relationship model. ACM Computing Surveys, 18(2):197-222, June 1986.

[UW02] J.D. Ullman and J. Widom. A First Course in Database Systems. Prentice Hall, Upper Saddle River, New Jersey, second edition edition, 2002. 